\title{
Barrier Evaluation by Linearly Increasing Voltage Technique Applied to Si Solar Cells and Irradiated Pin Diodes
}

\author{
E. Gaubas, T. Čeponis, V. Kalendra, J. Kusakovskij, and A. Uleckas \\ Institute of Applied Research, Vilnius University, Sauletekio Avenue 9-III, 10222 Vilnius, Lithuania \\ Correspondence should be addressed to E. Gaubas, eugenijus.gaubas@ff.vu.lt
}

Received 8 August 2011; Accepted 19 September 2011

Academic Editors: A. O. Neto and H. Saxén

Copyright () 2012 E. Gaubas et al. This is an open access article distributed under the Creative Commons Attribution License, which permits unrestricted use, distribution, and reproduction in any medium, provided the original work is properly cited.

\begin{abstract}
Technique for barrier evaluation by measurements of current transients induced by linearly increasing voltage pulse based on analysis of barrier and diffusion capacitance changes is presented. The components of the barrier capacitance charging and generation/recombination currents are discussed. Different situations of the impact of deep center defects on barrier and diffusion capacitance changes are analyzed. Basics of the profiling of layered junction structures using the presented technique are discussed. Instrumentation for implementation of this technique and for investigations of the steady-state bias infra-red illumination and temperature dependent variations of the barrier capacitance charging and generation/recombination currents are described. Applications of this technique for the analysis of barrier quality in solar cells and particle detectors fabricated on silicon material are demonstrated.
\end{abstract}

\section{Introduction}

Potential barrier in pin diodes and its stability under irradiations are the essential characteristics of particle detectors [1]. In the detectors heavily irradiated by high-energy particles, formation of the extended defects within a range of metallurgic junction is very probable $[2,3]$. Then junction interface circuitry shortening by microplasma generation and other metallurgic junction damage effects can appear. Similarly defects introduced by technological (e.g., doping) procedures are responsible for emerging of a deep level system within band gap of semiconducting materials. The deep levels aggravate the parameters of a junction and its operational characteristics. Scanning of the depth distribution of spreading resistance $\left(R_{S}\right)$ is a common and widespread tool for evaluation of the doping profiles within layered structures [4-8]. This method is well standardized for scanning spreading resistance microscopy (e.g., [5]), approaching to spatial resolution of a few $\mathrm{nm}$ [6]. It is often used for the technological control in formation of the device structures. However, doping and steady-state carrier density profiles can be insufficient to evaluate and to predict the operational characteristics of fabricated junctions if additional defects are introduced together with dopants. Thus, comprehensive techniques for testing of junction device structures are desirable.

In this work, a technique for barrier evaluation by linearly increasing voltage (BELIV) based on measurements of current transients at reverse and forward biasing is presented. A linear increment of the bias voltage is beneficial to reach small changes in depletion width with time. The latter technique is also preferential to have a constant ramp of the external voltage variation and of electric field-induced displacement current. Small and monotonic changes of depletion width are preferential to register the characteristic times of the fast thermal generation processes attributed to the rather shallow centers in the range of operation temperatures of devices under test and to identify the full depletion regime for heavily irradiated diodes. This technique has been tested on perfect diode structures and applied to analysis of barrier quality of Si detectors irradiated by reactor neutrons $(1 \mathrm{MeV}$ eq.) and protons with fluences in the range of $10^{12}-3 \times$ $10^{16} \mathrm{~cm}^{-2}$. Application of this barrier control technique, combined with a step-positioning of the needle-tip probe, as a rearward electrode, located on the cross-sectional boundary of a parallel-plate-layered structure is also approved for 


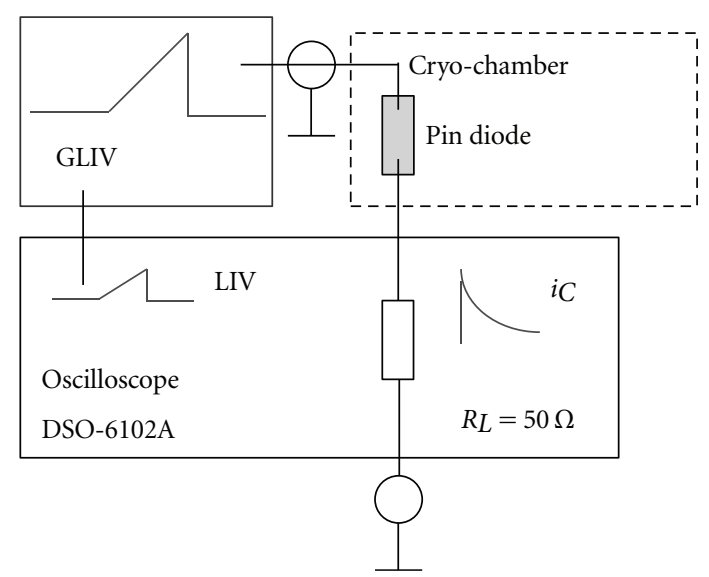

(a)

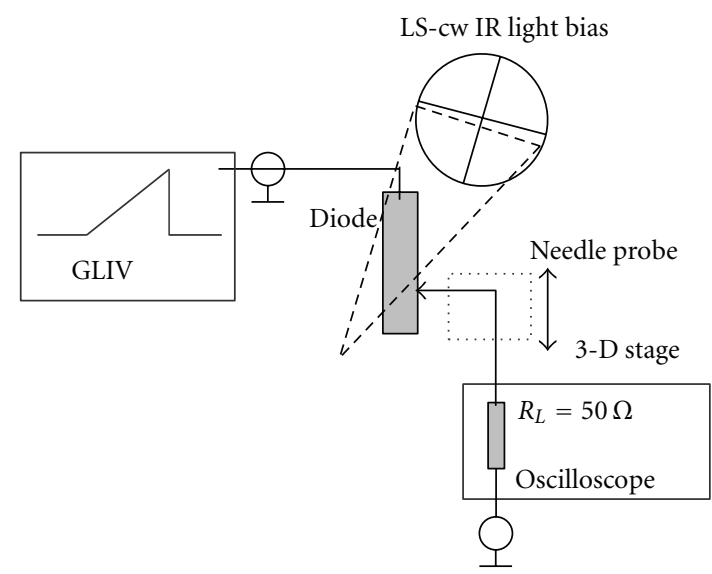

(b)

FIGURE 1: (a) Sketch of the measurement circuitry for implementation of the BELIV technique. GLIV generator of linearly increasing voltage. (b) Measurement circuitry for profiling of doping and defects density. LS is a continuous-wave infrared (IR) light source employed to vary expediently a filling of deep centers.

control of solar cells. The latter arrangement enables one to control spreading resistance, carrier injection efficiency and the parameters associated with barrier capacitance of a junction.

\section{Samples and Measurement Circuitry}

A set of pin diodes of CERN standard $p^{+} n n^{+}$detector structure (with dopants density $N_{D} \approx 10^{12} \mathrm{~cm}^{-3}$ in $n$-layer) [1] were investigated. Measurements of current transients were carried out in the range of temperatures from 120 to $300 \mathrm{~K}$. Several types of nonirradiated devices and materials have been also tested in approval of junction evaluation by BELIV technique.

Various $n^{+} p$ solar-cell fragments of different top layer thickness and of junction area have been examined. The resistivity of $p$-layer in solar-cells significantly exceeds that of high resistivity layer within pin diodes. Variations of the peak amplitudes have been examined in order to resolve an interface location within an $n^{+} p$ junction structure.

A sketch of measurement circuitry for implementation of different BELIV regimes is illustrated in Figure 1. The measurement circuitry contains an adjusted output of a generator of linearly increasing voltage (GLIV), a diode under investigation, and a load resistor connected in series. Current transients have been registered using a $50 \Omega$ external resistor or load input of the Agilent Technologies DSO6102A oscilloscope. The other channel of the digital oscilloscope is exploited for synchronous control of linearity of a GLIV signal using a signal differentiating procedure installed within DSO oscilloscope. Linearity of the GLIV signal is essential within implementations of the BELIV technique; therefore, several types of generators were tested to get suitable LIV characteristics.

The diode under test is mounted on a cold finger within a vacuumed cryo-chamber for examination of the temperature variations of BELIV characteristics, as sketched in Figure 1(a).
Arrangement for the BELIV profiling is sketched in Figure 1(b). The cross-sectional boundary of a layered junction structure is scanned by a gold needle positioned by the precise 3D stepper. The microstructure of a needle tip determines an appearance of the spreading currents between the main plate electrode of a relatively large area and the needle tip on a boundary of a structure located (perpendicularly to the main plate electrode) within a definite layer and at definite depth point. The boundary of the samples for depth profiling was carefully prepared to be as smooth as possible. A complementary continuous-wave infrared (IR) light source is employed to vary expediently a filling of deep centers.

\section{Analysis of Transients in Reverse and Forward LIV Biased Diodes for Evaluation of Barrier Parameters}

\subsection{Charge Extraction BELIV Regime}

3.1.1. Trap Free Material. The BELIV technique for a reverse biased diode is based on analysis of the changes of barrier capacitance $\left(C_{b}\right)$ with linearly increasing voltage $U_{p}(t)=A t$ pulse. The $C_{b}(t)$ dependence on voltage $U_{p}(t)$ and thereby on time $t$ can be described using the depletion approximation [8], for charge extraction in the trap-free material. This approximation for an abrupt $p^{+}-n$ junction in diode leads to a simple relation $C_{b}=C_{b 0}\left(1+U / U_{b i}\right)^{-1 / 2}$, where barrier capacitance for a nonbiased diode of an area $S$ is $C_{b 0}=$ $\varepsilon \varepsilon_{0} S / w_{0}=\left(\varepsilon \varepsilon_{0} S^{2} e N_{D} / 2 U_{b i}\right)^{1 / 2}$. The other symbols represent the following: $\varepsilon_{0}$ is a vacuum permittivity, $\varepsilon$ material dielectric permittivity, $e$ elementary charge, $U_{b i}$ built-in potential barrier, $w_{0}=\left(2 \varepsilon \varepsilon_{0} U_{b i} / e N_{D}\right)^{1 / 2}$ width of depletion for the non-biased junction, and $A=U_{P} / \tau_{P L}$ ramp of LIV pulse with $U_{P}$ peak amplitude and $\tau_{P L}$ duration. 
The depletion approximation [8] seems to be a rather good model for description of the charge extraction transients in time scale $t$ longer than the dielectric relaxation $\tau_{M}=\varepsilon \varepsilon_{0} / e \mu n_{0}$ time, when employing LIV pulses of durations $\tau_{P L}>\tau_{M}$. Here, it is assumed that an equilibrium carrier density $n_{0}$ is equal to the effective doping density $\left(n_{0}=\right.$ $\left.N_{\text {Deff }}\right)$. This is maintained by a short Debye length $L_{D}=$ $\left(2 \varepsilon \varepsilon_{0} k T / e^{2} n_{0}\right)^{1 / 2}$ relatively to the characteristic depletion widths $\left(w_{0}, w=w_{0}\left(1+U / U_{b i}\right)^{1 / 2}\right)$ and a geometric thickness $d\left(L_{D} \ll w_{0}, w, d\right)$ for $N_{\text {Deff }}>10^{11} \mathrm{~cm}^{-3}$.

The time-dependent changes of charge $q=C_{b} U$ within junction determine a current transient $i_{C}(t)$ in the external circuit:

$$
\begin{aligned}
i_{C}(t)= & \frac{d q}{d t} \\
& =\left\{\begin{array}{l}
\frac{\partial U}{\partial t}\left(C_{b}+U \frac{\partial C_{b}}{\partial U}\right)=\frac{\partial U_{C}}{\partial t} C_{b 0} \frac{1+\left(U_{C}(t) / 2 U_{b i}\right)}{\left(1+\left(U_{C}(t) / U_{b i}\right)\right)^{3 / 2}} \\
\approx A C_{b 0} \frac{1+\left(A t / 2 U_{b i}\right)}{\left(1+\left(A t / U_{b i}\right)\right)^{3 / 2}}, \\
\text { at } U<U_{\mathrm{FD}}, \\
\frac{\partial U}{\partial t}\left(C_{\text {geom }}+U \frac{\partial C_{\text {geom }}}{\partial U}\right)=\frac{\partial U_{C}}{\partial t} C_{\text {geom }} \approx A C_{\text {geom }}, \\
\text { for } U \geq U_{\mathrm{FD}} .
\end{array}\right.
\end{aligned}
$$

This transient for a rather small peak voltage $U_{P}$ below values $U_{P}<U_{\mathrm{FD}}$ of full depletion voltage $\left(U_{\mathrm{FD}}\right)$ contains an initial $(t=0)$ step $A C_{b 0}$ due to displacement current and a descending component governed by the charge extraction. For a strongly compensated material and for $U_{P}>U_{\mathrm{FD}}$, this transient contains only a displacement current step. When parameters of material and of LIV pulse match a condition $0<U_{P} \leq U_{\mathrm{FD}}$, the transient contains a displacement current step and a descending charge extraction component, which saturates at time instant $t_{\mathrm{FD}}=U_{\mathrm{FD}} / A+C_{\text {geom }} R_{L}$. The descending component of charge extraction for $U_{P}<U_{\mathrm{FD}}$, gives an additional relation to extract $U_{b i}$ by taking a ratio $r_{m}=i_{C}(0) / i_{C}\left(t_{m}\right) \geq 1$ at fixed time instant $t_{m}$. Actually, a real positive root of the cubic equation $\left(A / U_{b i}\right)^{3} t_{m}{ }^{3}+(3-$ $\left.r_{m}^{2} / 4\right)\left(A / U_{b i}\right)^{2} t_{m}^{2}+\left(A / U_{b i}\right)\left[3-r_{m}^{2}\right] t_{m}+\left(1-r_{m}^{2}\right)=0$ should be found. Viete's relation for a product of roots of the cubic equation and Routh-Hurwitz' criteria (for $\left(1-r_{m}{ }^{2}\right)<$ 0 ) indicate that such a root exists. Subsequently value of $N_{D}$ is evaluated by substituting the extracted $U_{b i}$ within the initial current expression $i_{C}(0)=A C_{b 0}$. Having determined $N_{D}$, the density of acceptors $N_{A}$ in $p^{+}$layer can be verified by using a well-known relation $U_{b i}=(k T / e) \ln \left(N_{A} N_{D} / n_{i}^{2}\right)$ with intrinsic carrier density $n_{i}$. Therefore, the mentioned procedure, using (1), can be employed for primary analysis of a fragment of transient (in perfect junction structures), experimentally measured for a fixed LIV pulse duration $\tau_{P L}$. The experimental BELIV transients, measured on a nonirradiated diode and shown in Figure 2, are in agreement with diode current transients approximated by (1). The vertex amplitude (Figure 2) within a transient is proportional to a value of a ramp $A$ of LIV pulse, which is controlled by

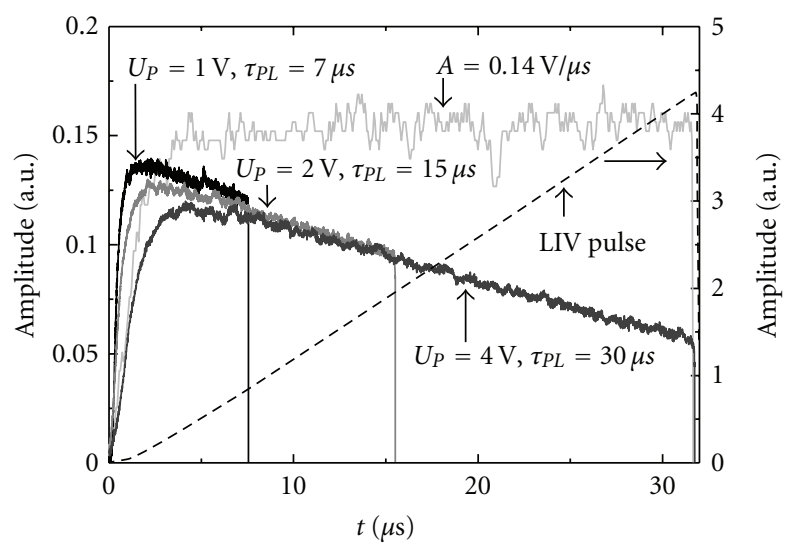

FIgure 2: Transients of the charge extraction current measured in the commercial diode at a reverse $\left(U_{R}\right)$ polarity of LIV pulses of varied duration keeping a constant LIV ramp.

differentiating $\left(d U_{p} / d t\right)=A$ the LIV pulse $\left(U_{p}(t)\right)$, as illustrated in Figure 2. The simulated (using (1)) BELIV current transient is illustrated by solid curve in Figure 3(a). To extract the barrier parameters more precisely, the above mentioned procedures can be applied after diffusion current is evaluated. The latter may be a reason in formation of a pedestal for $i_{C}(t)$ changes.

Diffusion current is a rapidly stabilized (for $t>k_{B} T / e A$ ) function $i_{\text {diff }}(t)=i_{\text {diffo }}\left[1-\exp \left(-e A t / k_{B} T\right)\right] \cong e S n_{i}^{2} L_{D p} /$ $N_{D} \tau_{p}\left[1-\exp \left(-e A t / k_{B} T\right)\right] \approx e S n_{i}^{2} L_{D p} / N_{D} \tau_{p}$ at reverse biasing. Here, additional symbols represent the following: $k_{B} T$ is thermal energy at temperature $T ; L_{n, p}=\left(D_{n, p} \tau_{n, p}\right)^{1 / 2}$ is a diffusion length for electrons $(n)$ and holes $(p)$ in $p$ and $n$ layers of a diode, respectively. The stabilized value of $i_{\text {diff }}\left(t \gg k_{B} T / e A\right)=i_{\text {diffo }}$ can be ignored in comparison with $i_{C}(t)$ for properly fabricated (containing low density of traps and large $L_{n, p}$ ) diodes in the realistic range of LIV pulse durations. The diffusion current in the range of $t \leq k_{B} T / e A$ determines a differential resistance of a junction, which may limit an increase of $i_{C}(t \approx 0)$. Really, a delay appears due to serial processes of dielectric relaxation within quasi-neutral range of nondepleted n-layer, drift, and diffusion of carriers to complete a circuit. The initial delay is caused by the characteristic times of diffusion $\tau_{D}=L_{D}{ }^{2} / 2 D$ and of dielectric relaxation $\tau_{M}$. The mentioned characteristic lifetimes, summarized as $\tau_{R C}$, may comprise a delay. Additional $R C$ appears due to the external circuit and the initial nonlinearity of GLIV pulse. The latter can be noticed as a deviation from a square-wave shape pulse within very initial stages of the differentiated experimental GLIV pulse.

The BELIV transients for the abrupt junction diodes have been discussed above. Similarly, for a linearly graded ( $\mathrm{Lg}$ ) junction, the current $i_{C}(t)$, due to depletion of Lg junction, can be expressed as $i_{C \mathrm{Lg}}(t)=A C_{b 0 \mathrm{Lg}}\left(1+2 A t / 3 U_{b i \mathrm{Lg}}\right) /$ $\left(1+A t / U_{b i \mathrm{Lg}}\right)^{4 / 3}$. Here, the rearranged parameters of $U_{b i \mathrm{Lg}}$, $w_{0 \mathrm{Lg}}$, and $C_{b 0 \mathrm{Lg}}$, as for example, in [9], should be used.

3.2. Impact of the External Circuit. The load resistor $R_{L}$, as a linear circuit component for registration of the BELIV 


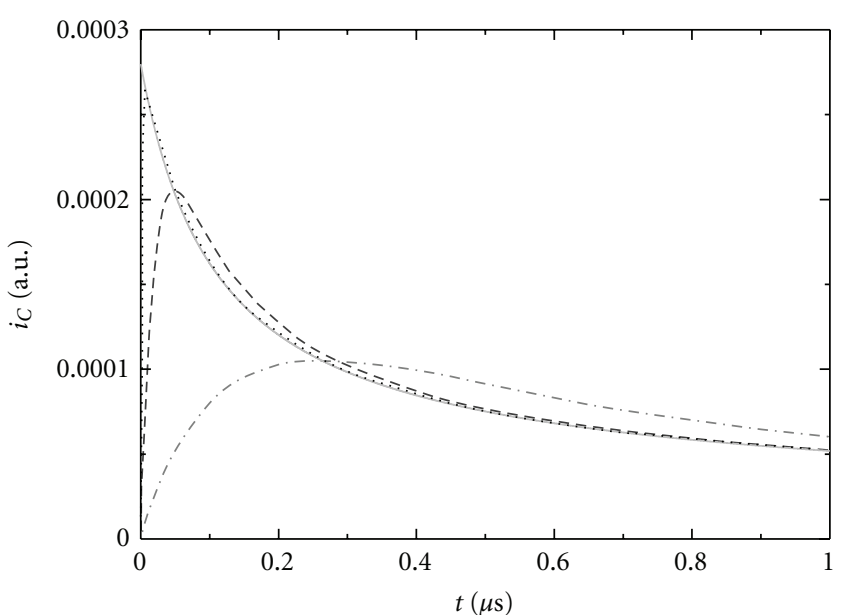

$\begin{aligned} R C & =0 \mathrm{~ns} \\ \ldots . & R C=2 \mathrm{~ns}\end{aligned}$

(a)

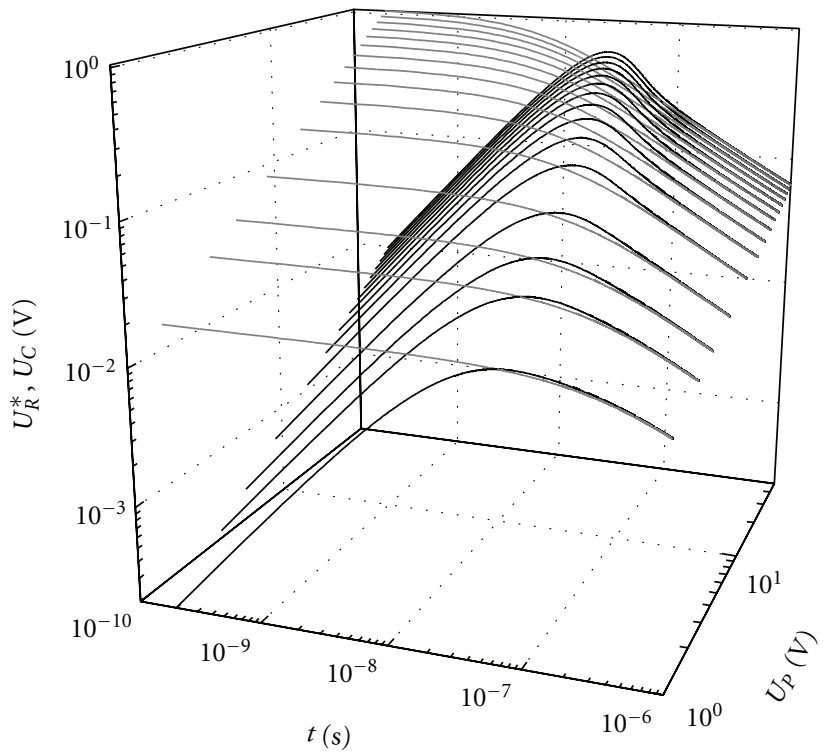

$-U_{R}^{*}$

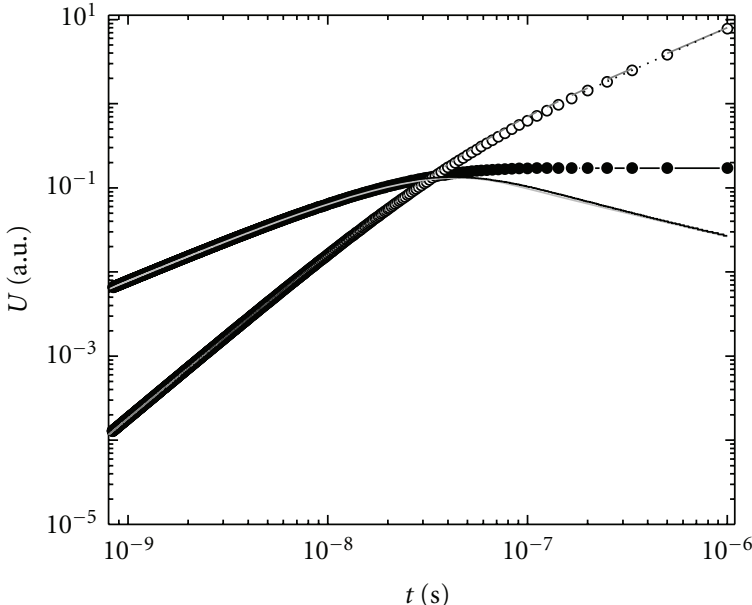

$-\bullet-U_{R}, C=$ const $\quad-U_{C=C(t)}$

.... $U_{C}=$ const

$-U_{R}, C=C(t)$

- $U_{R}$ analytic BELIV model (b)

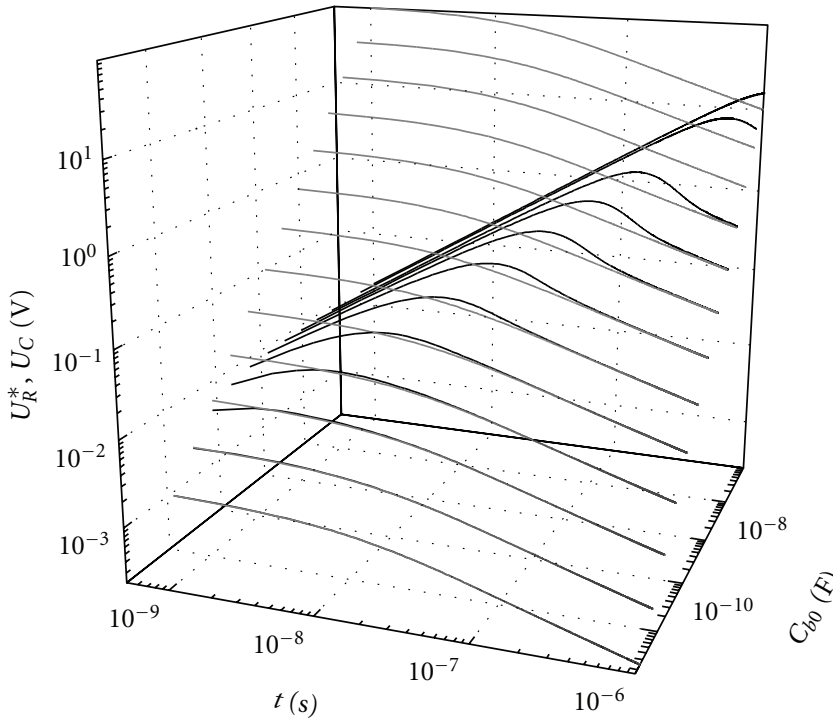

$-U_{R}^{*}$

(c)

(d)

FIgURE 3: (a) Simulated BELIV currents $i_{C}(t)$ for a diode with $C_{b 0}=70 \mathrm{pF}$, calculated without delay (solid curve) and using convolution integral with $R C$ values of $R C=2 \mathrm{~ns}$ (dot), $R C=20 \mathrm{~ns}$ (dash), and $R C=200 \mathrm{~ns}$ (dash-dot). (b) Simulated time-dependent voltage drops on load resistor $\left(R_{L}=50 \Omega\right)$ and on capacitor $(C=430 \mathrm{pF})$ for LIV pulse of $\tau_{P L}=1 \mu$ s of a peak voltage $U_{P}=8 \mathrm{~V}$ and the BELIV transients simulated by using (1) and (2) ( black symbol + line), by using (1) and (4) (gray line), and analytic solutions with $R C=20 \mathrm{~ns}(1)$ and (3) (black line). (c) Numerically simulated BELIV voltage transients $U_{R}{ }^{*}(t)$ as a function of an LIV pulse peak voltage $U_{P}$ compared with those simulated by analytical approximation. (d) Numerically simulated BELIV voltage transients $U_{R}{ }^{*}(t)$ as a function of initial barrier capacitance values $C_{b 0}$ compared with those simulated by analytical approximation.

current transient, determines the time-dependent voltage sharing in $R C$ circuit between the $R_{L}$ and the diode with barrier capacitance $C_{\mathrm{b} 0}$.

The solution of a simple differential equation $R_{L} d i / d t+$ $(1 / C) i=A$, derived for the linear elements, as a capacitor $C$ and an $R_{L}=R$ connected in serial, biased by a LIV pulse
$U=A t$, and using an initial condition of $i(t=0)=0$, leads to the following expressions:

$$
\begin{aligned}
i(t) & =A C\left(1-e^{-t / R C}\right), \\
U_{R}(t) & =A C R\left(1-e^{-t / R C}\right)=\left\{\begin{array}{l}
\left.A t\right|_{t \ll R C,}, \\
\left.A C R\right|_{t \gg R C},
\end{array}\right.
\end{aligned}
$$




$$
\begin{aligned}
U_{C}(t) & =A\left[t-R C\left(1-e^{-t / R C}\right)\right] \\
& =\left\{\begin{array}{l}
\left.A \frac{t^{2}}{2 R C}\right|_{t \ll R C} \\
\left.A(t-R C) \cong A t\right|_{t \gg R C}
\end{array}\right.
\end{aligned}
$$

Equation (2) implies that the linear voltage drop on a capacitor (and on a diode under test-DUT) appears for time instants $t \gg R C$. The maximal barrier capacitance $C_{b 0}$ for DUT acts during the initial instants of LIV pulse (when $A t<$ $\left.U_{b i}\right)$. Thus, the fastest initial component of the BELIV current transient is determined by the transition time constant $R C_{b 0}$. The linear $R C_{b 0}$ modifications (e.g., a shift) of the initial current step $i_{C}(t \approx 0)$ in (1) can be roughly emulated by a convolution integral:

$$
i_{R C}(t)=\frac{1}{\tau_{R C}} \int_{0}^{t} i_{C}(x) \exp \left[-\frac{(t-x)}{\tau_{R C}}\right] d x,
$$

shown by the broken curves in Figure 3(a). Then, the peak within a current transient appears on the $i_{C}(t)$ curve, simulated by (1) (a dot curve in Figure 3(a)). A kink (at $t \approx R C$ ) within the initial rise front of the simulated BELIV transient and a peak position of the experimental one might be employed for the evaluation of the $C_{b 0}$ using a rough linear approximation (by a convolution integral) of a BELIV transient.

However, variation of the voltage drop $U_{C}(t)=A(t-$ $R C(t)$ ) on a nonlinear DUT can be assumed being a linear function of $t$ only in time scale $t>2 R C$ (2). For comparison, the simulated (using (2)) time-dependent voltage drops on the load resistor (dot curve) and on a capacitor (solid curve) are illustrated in Figure 3(b). It is worth noting, that, for a dielectric capacitor within $R C$ circuit, the voltage response, measured on the load resistor using LIV, is close to a squarewave pulse (dot curve, Figure 3(b)).

For the precise description of BELIV transient, a generalized differential equation

$$
\frac{d U_{C}(t)}{d t} \frac{\left(1+U_{C}(t) / 2 U_{b i}\right)}{\left(1+U_{C}(t) / U_{b i}\right)^{3 / 2}}-\frac{U_{p}(t)-U_{C}(t)}{R C_{b 0}}=0
$$

with the initial conditions $U_{p}(t=0)=0$ and $U_{c}(t=0)=0$ should be solved to determine $U_{C}(t)$. Equation (4) is derived assuming the time-dependent voltage drops on DUT, as $U_{C}(t)=U_{p}(t)-U_{R}(t)$. So (4) is a non-linear differential equation, more complicated than a Riccati' one. Only the numerical solutions of the (4) can be obtained. Using a solution $U_{C}{ }^{*}(t)$ of $(4)$, a voltage drop on the load resistor, as $U_{R}{ }^{*}(t)=U_{p}(t)-U_{C}{ }^{*}(t)$, represents the simulated BELIV transient. Thus, a fitting procedure, of the numerically simulated BELIV transients $U_{R}{ }^{*}(t)$ to the experimental ones, is inevitable and has been employed in this work for precise extraction of the junction parameters.

Comparisons of the simulated voltage transients (using $U_{R}{ }^{*}(t)$ obtained by solution of (4), and using $U_{C}(t)(2)$ values evaluated for fixed $C$ using analytical approximations
(Eqs. (1) and (3)) for $R C=20 \mathrm{ns)}$ ) are presented as a function of LIV pulse peak voltage $U_{P}$ and as a function of value of the equilibrium barrier capacitance $C_{b 0}$ in Figures 3(c) and 3(d), respectively. It can be noticed in Figures 3(c) and 3 (d) that a deviation from the analytically simulated curves appears in the range of the BELIV voltage peak. This deviation increases with increment of $U_{P}$ and of $C_{b 0}$ values. These deviations can be explained by the relative enhancement of barrier charging current through the load resistor. This current increase modifies non-linearly the voltage drops (as included in (4)) on a load resistor and on a diode. Thus, the analytical approximation (1), (2), and (3) can be exploited for primary analysis of the BELIV transients only in the range of small $i_{C}$ currents, and including $R C$ shift.

3.3. BELIV Transients in Traps Containing Material. The variety of processes and effects, determined by different ratios of the characteristic times of carrier capture as well as of carrier thermal emission ascribed to deep traps, and of dielectric relaxation, responsible for a stabilized depletion boundary, is rather wide [8], especially when carrier redistribution among several centers takes place. Here, an impact of only a few of them is briefly discussed.

3.3.1. Generation Centers. The radiation traps are responsible for generation current within depletion region. Depending on carrier emission $\left(\tau_{e m}\right)$ and capture $\left(\tau_{c p}\right)$ characteristic times (when $\tau_{e m}>\tau_{c p}>\tau_{P L}$ ) a definite density of traps can persist filling for a rather long time-gap relatively to a LIV pulse duration.

Generation centers can be observable in BELIV current response either by modification of a depletion width (by changing of the applied electric field distribution) during an LIV pulse or by collected charge, when increment of depletion width (bulk) highlights an impact of the generation current. The prevailing regime can be resolved only in the experiments.

Modification of the depletion width (when generation centers are able to redistribute electric field within a depletion area) can appear through changes in the built-in potential as $U_{b i}=\left(k_{B} T / e\right)\left\{\ln \left(N_{A} N_{D} / n_{i}{ }^{2}\right)+\ln \left[1-\left(N_{A n} \pm\left(N_{T}{ }^{ \pm}-\right.\right.\right.\right.$ $\left.\left.\left.\left.n_{T}(t)\right)\right) / N_{D}\right]\right\}$, where $N_{D}$ is a doping density of the nonirradiated material and $N_{A n}$ is a density of radiation-induced acceptors in the $n$-type material within the lower half of band gap. Also, there are traps $N_{T}{ }^{ \pm}$of acceptor (+) or donor-type $(-)$ within the upper-half of the band gap with respective temporal their filling $n_{T}(t)$ (here, superscript $(+/-)$ shows selection of the sign before bracket, not electrical charge). Thus, the built-in barrier has two components $U_{b i}(t)=$ $U_{b i 0}+\left(k_{B} T / e\right)\left\{\ln \left[1-\left(N_{A n} \pm\left(N_{T}^{ \pm}-n_{T}(t)\right)\right) / N_{D}\right]\right\}$. The second component can be modulated by LIV, when the duration of the characteristic emission/capture processes approaches to a dielectric relaxation $\left(\tau_{M}\right)$ time, that is, $\tau_{c p} \approx \tau_{e m} \sim \tau_{M}$. Also a component in the temporal modulation of the depletion width $w(t)$ appears due to changes in the effective doping as $N_{\text {Deff }}(t)=N_{D} \times\left[1-\left(N_{A n} \pm\left(N_{T}^{ \pm}-n_{T}(t)\right)\right) / N_{D}\right]$. 
These fast trap filling variations result in temporal changes of barrier capacitance as

$$
\begin{aligned}
C(t)= & C_{b 0} \times\left(1-\frac{\left(N_{A n} \pm\left(N_{T}^{ \pm}-n_{T}(t)\right)\right)}{N_{D}}\right)^{\frac{1}{2}} \\
& \times\left\{1+U_{b i 0}^{-1}\right. \\
& \left.\times\left(U(t)+\frac{k_{B} T}{e} \ln \left[1-\frac{N_{A n} \pm\left(N_{T}^{ \pm}-n_{T}(t)\right)}{N_{D}}\right]\right)\right\}^{-\frac{1}{2}} .
\end{aligned}
$$

Here, $C_{b 0}$ is expressed by the parameters of the nonirradiated diode. Then, the BELIV current can be derived as a time-differentiated response $i(t)=\left(d U_{C} / d t\right) \times\{C(t)+$ $\left.U_{C}(t)\left[(d C / d w)\left(d w / d U_{C}\right)+(d C / d w)\left(d w / d n_{T}\right)\left(d n_{T} / d U_{C}\right)\right]\right\}$. However, this leads to very cumbersome expressions. The simulated BELIV current transient, using the approach of fast traps $\left(\tau_{c p} \approx \tau_{e m} \sim \tau_{M}\right)$, is compared in Figure 4 with that for a diode without traps, when keeping the same values of other parameters. The simulated transient shows an impact of carrier generation current in the ulterior stages of a transient by exceeding those current values for a diode without traps. The main point of reference for separation of this trapmodulated BELIV regime is a coincidence of current values within initial stage of transients for a fixed LIV pulse ramp $d U_{C} / d t$ although the simulated transients appear to be considerably complicated when variations of the parameters of $N_{A n}, N_{T}{ }^{ \pm}$, and $n_{T}(t)$ are involved. It is worth noting that this regime should be more pronounced when a single type (fast) trap dominates.

For charge collection BELIV regime, the generation current is included by a simple increase of volume from which carriers are collected by increased depletion width, during LIV pulse evolution. The generation current $i_{g}(t)=$ $e n_{i} S w_{0}\left(1+U_{C}(t) / U_{b i}\right)^{1 / 2} / \tau_{g}$ increases with voltage $U_{C}(t)$ and can exceed the barrier charging current in the rearward phase of the transient. Here, $N_{D}$ in expressions for $w_{0}$ and $C_{b 0}$ should be replaced by its effective value $N_{\text {Def }}=N_{D} \pm N_{T}{ }^{ \pm}$, due to (compensation) charged traps of density $N_{T}$. Then, transient of the total reverse current is described by a sum of the currents:

$$
\begin{aligned}
i_{R \Sigma}(t)= & i_{C}(t)+i_{\text {diff }}(t)+i_{g}(t) \\
= & A C_{b 0} \frac{1+U_{C}(t) / 2 U_{b i}}{\left(1+U_{C}(t) / U_{b i}\right)^{3 / 2}} \\
& +i_{\text {diffo }}\left(1-e^{-\left(e U_{C}(t)\right) /\left(k_{B} T\right)}\right) \\
& +\frac{e n_{i} S w_{0}}{\tau_{g}}\left(1+\frac{U_{C}(t)}{U_{b i}}\right)^{1 / 2} .
\end{aligned}
$$

The descending charge extraction component and the ascending generation current component imply existence of a current minimum within a current transient (approximated by (1)). The time instant $t_{e}$, for which this extremum appears, is determined by using a condition $d i_{R \Sigma} /\left.d t\right|_{t e}=0$.

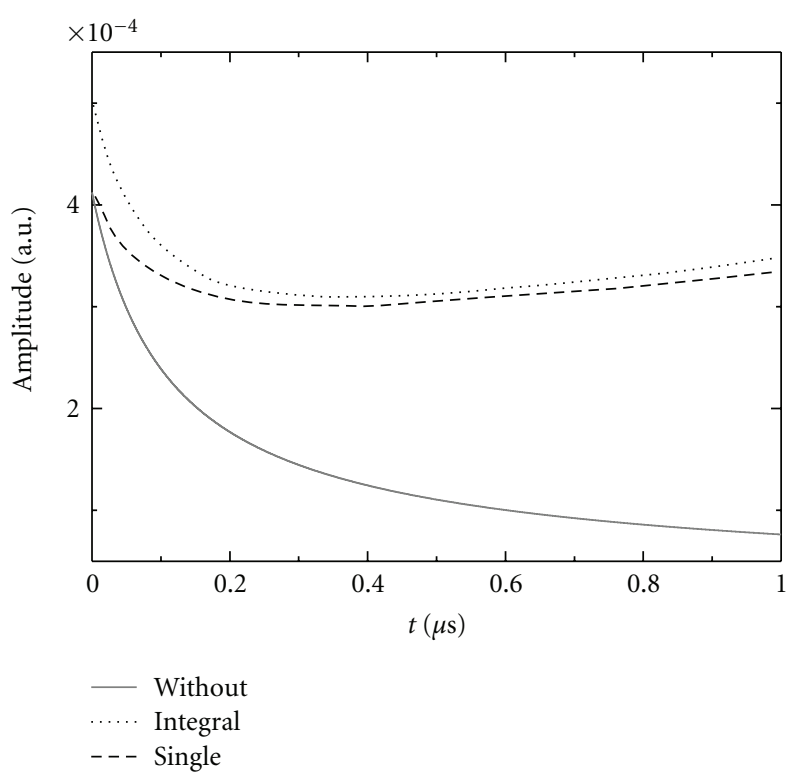

FIGURE 4: Comparison of charge extraction BELIV current transients simulated for single-type traps (dash) and for simultaneously acting several-type generation centers (dots) with that simulated without contribution of thermal emission from traps (solid curve).

This leads (at assumption $d i_{\text {diff }}(t) / d t=0$ for $t_{e} \gg$ $\left.k_{B} T / e\left(d U_{c}(t) / d t\right)\right)$ to a relation for the peculiar time instant $t_{e}\left(\right.$ for $\left.i_{\Sigma}(t)=\min \right)$, as

$$
\begin{aligned}
t_{e}= & \frac{U_{b i}}{\left(d U_{\mathrm{c}} / d t\right) i_{g}(0)} \\
& \times\left[\frac{i_{C}(t)}{4}-i_{g}(0)+\sqrt{\left(\left(\frac{i_{c}(t)}{4}\right)^{2}+\frac{3}{2} i_{c}(t)\right) i_{g}(0)}\right] .
\end{aligned}
$$

The initial component of the composite current $\left(i_{R \Sigma}(t) \approx\right.$ $i_{C}(t)+i_{\text {diff }}(t) \gg i_{g}(0)$ for $\left.t \ll t_{e}\right)$ can be exploited for evaluation of the barrier $U_{b i}$ height. Here, value of $i_{\text {diffo }}$ determined for a nonirradiated diode can be employed for separation of the current components. Subsequently, carrier generation lifetime can be evaluated by using (7). The simulated current transient for the latter BELIV (charge collection) regime is illustrated in Figure 4. Also, the peculiar points are denoted in Figure 4. The main point of reference, to identify this charge collection BELIV regime, is an increment of current value relatively to that for the nonirradiated sample at fixed LIV pulse ramp $d U_{C} / d t$. This regime is the most probable one when several traps (primary filled) of different species simultaneously emit carriers.

For the heavily irradiated diodes, nearly all the "native" carriers $\left(n_{0}=N_{D}\right)$ are captured by $N_{T}$ traps. Capture of $n_{0}$ carriers leads to a full depletion regime. In the fully depleted diode, enhancement of applied voltage determines a shortening of the time of carrier transit $\tau_{t r}=d^{2} /\left.\mu U_{C}\right|_{\mathrm{FD}}$ across the interelectrode gap equal to sample thickness $d$, approaching to relation $\tau_{t r} \leq \tau_{e m}$. In diode biased above full depletion voltages, the total BELIV current can be described (similarly 
to the method used in [8]) by considering conductivity and displacement current components. This consideration is performed for variable voltage and for surface charge changes on electrodes, at standard boundary conditions (for electric field $\left.E\right|_{x=d}=0, d E /\left.d x\right|_{x=d}=0$ and for potential $\left.V\right|_{x=d}=0$ ). This solution is expressed and approximated (for $\tau_{t r} \sim \tau_{\text {em }}$ and $n \approx n_{0}=N_{D}$ ), as

$$
\begin{aligned}
\left.i(t)\right|_{F D} & =e S \frac{d}{2}\left(\frac{d n}{d t}+\frac{d p}{d t}\right)+\frac{\varepsilon \varepsilon_{0} S}{d} \frac{d U_{C}}{d t} \\
& \approx e S \frac{d}{2} \frac{n_{0}}{\tau_{t r}}+C_{\text {geom }} \frac{d U_{C}}{d t} \\
& =e \frac{S}{2 d} n_{0} \mu_{n} U_{C}(t)+C_{\text {geom }} \frac{d U_{C}(t)}{d t} .
\end{aligned}
$$

Here, $C_{\text {geom }}=\varepsilon_{0} \varepsilon S / d$ is a geometrical capacitance. It is clear that for trap-free insulating material (when $d(n, p) / d t=0$ ) the BELIV current transient $i_{\mathrm{CFD}}=C_{\text {geom }}\left(d U_{C} / d t\right)$ acquires a shape of square-wave pulse, at voltages $\left.U_{C}\right|_{F D}$. For the trapsrich (compensated) material, further increment of voltage above $U_{\mathrm{FD}}$ leads to the increase of current component $i_{\text {emFD }}(t)=e(S / 2 d) n_{0} \mu_{n} U_{C}(t)$, added to an $i_{\mathrm{CFD}}$.

To resolve the prevailing BELIV regime, the external factors (e.g., steady-state light biasing) can be employed. These factors modify the occupation of the trap states and highlight the dominant components of current.

3.3.2. Carrier Capture Centers. In a reverse-biased diode, the carrier capture process is probable and makes the main impact either within a Debye screening length (for a steady state) or during a dielectric relaxation time $\tau_{M}$ within a transition distance $\lambda=\left[2 \varepsilon_{0} \varepsilon\left(E_{F}-E_{T}(\lambda) / e^{2} N_{D}\right]^{1 / 2}\right.$ at a depletion boundary [8]. The transition distance $\lambda$ defines a length at which flat band condition (going from the depletion region, that is, band bending side) for a definite centre $E_{T C}$ is achieved. This is reached by a voltage drop $\Delta U_{\lambda}=\left(E_{F}-\right.$ $\left.E_{T}(\lambda)\right) / e$ on a $\lambda$ thick depletion layer close to the interface with electrically neutral material region. This voltage drop $\Delta U_{\lambda}$ positions a trap level below a Fermi level (trap is occupied and neutral). The mentioned definition of $\lambda$ can be rearranged to a condition within a scale of characteristic times, as $\tau_{M}=\tau_{t r \lambda} / 2$, with $\tau_{M}=\varepsilon \varepsilon_{0} / e \mu n_{0}$ and $\tau_{t r \lambda}=$ $\lambda^{2} / \mu \Delta U_{\lambda}$. The latter condition tells that $\lambda$ is a distance for which dielectric relaxation time should be a half of the transition time $\left(\tau_{t r \lambda} / 2\right)$. This result can be understood by the interplay of conductivity and displacement current components within a total current (like the first component $(d / 2)$ of a sum in (8), when only a half of the conductivity current flows towards external circuit). On the other hand, screening of the depletion field within $\lambda$ can be reached by extraction of thermally emitted carriers from the depleted layer and by free carriers diffused from the neutral material side,- - this shortens a relaxation time twice. Recombination or capture of carriers within $\lambda$ layer modifies this screening condition as $\tau_{M}=\left[2 / \tau_{\text {tr } \lambda}+1 / \tau_{R}\right]^{-1}$. Thus, transit time is modified due to carrier capture within $\lambda$ layer, as the carrier diffusion length (from a neutral material side) is reduced (relatively to that of $\tau_{R} \rightarrow \infty$ ). Then dielectric relaxation time (during which an impact of free carriers on space charge and electric field vanishes at a depletion boundary) approaches to the carrier capture time $\left(\tau_{M} \rightarrow \tau_{c p, R}\right)$, when the transit time becomes significantly longer than that of capture/recombination $\left(\tau_{c p, R}\right)$ one. In fully depleted (insulating) material, the carrier capture and recombination processes are essential nearby the electrodes, although processes are of the same origin.

In material containing high density of wide spectrum of deep traps $N_{T}$, current $i_{c p}(t)$ generated by GLIV flows to fill the traps. Then, position of Fermi quasi-level varies within band-bending $\lambda$ layer of the depletion range. The current response (due to carrier capture within $\lambda$ layer in time-scale of a LIV pulse) can be expressed as

$$
i_{c p}(t)=\frac{e n_{0}\left(t, \tau_{M}, \tau_{t r \lambda}\right) S \lambda}{\tau_{c p}},
$$

when several species of deep centers are involved. Alternatively, carrier recombination/capture processes can be considered through capture rate $u(t)$ (accepted in [8] as the socalled extended depletion approximation). Carrier capture actually modifies the doping $N_{D}(t)$ density capable to ensure the barrier, $N_{D}(t)=N_{\text {Def }} u(t)$. For high density of carrier capture centers, an amplitude of response is nearly proportional to the current $i_{c p}(t)$. This carrier capture $(u<1)$ response saturates and approaches to value $u\left(t>\tau_{c p}\right)=1$ for time instants exceeding carrier capture time, that is, when all the carrier capture centers are filled. For relatively long LIV pulses, with $\tau_{P L} \gg \tau_{c p}$ and with small value of $\operatorname{ramp} A$, an external current is insufficient to fill traps during the initial stages of the LIV pulse. Therefore, the BELIV current transient has no vertex in such a situation. The simulated BELIV current transients, using approximations involved within derivation of (3) and (9), are illustrated in Figure 5.

The simulated BELIV current transients indicate that clear barrier capacitance (initial peak due to barrier capacitance charging current) can be observed when trap density is lower than density of dopants. Only ascending current component can be observed for trap density higher than that of dopants.

3.4. Charge Injection BELIV Regime. Barrier current transients and their analysis become more complicated at forward biasing of a diode (a regime of minority carrier injection). Then, voltage drop on a load resistor should be always included: $U_{F}(t)=A t-R_{L} i_{\Sigma F}(t)$, to avoid phantom roots and poles within model equations. Generally, current appears to be composed of the barrier $\left(i_{C F}(t)\right)$ and of the diffusion (storage) capacitance charging $\left(i_{\text {Cdiff }}(t)\right)$ currents and of recombination $\left(i_{R C}(t)\right)$ and injection/diffusion $\left(i_{\text {injc } F}(t)\right.$, $\left.i_{\text {difF }}(t)\right)$ ones, as

$$
\begin{aligned}
& i_{\Sigma F}(t) \\
& =i_{C F}(t)+i_{\text {Cdiff }}(t)+i_{R}(t)+i_{\text {injcF }}(t) \\
& =\frac{\partial U_{F}(t)}{\partial t}\left[C_{b 0} \frac{\left(1-U_{F}(t) / 2 U_{b i}\right)}{\left(1-\frac{U_{F}(t)}{U_{b i}}\right)^{3 / 2}}\right.
\end{aligned}
$$




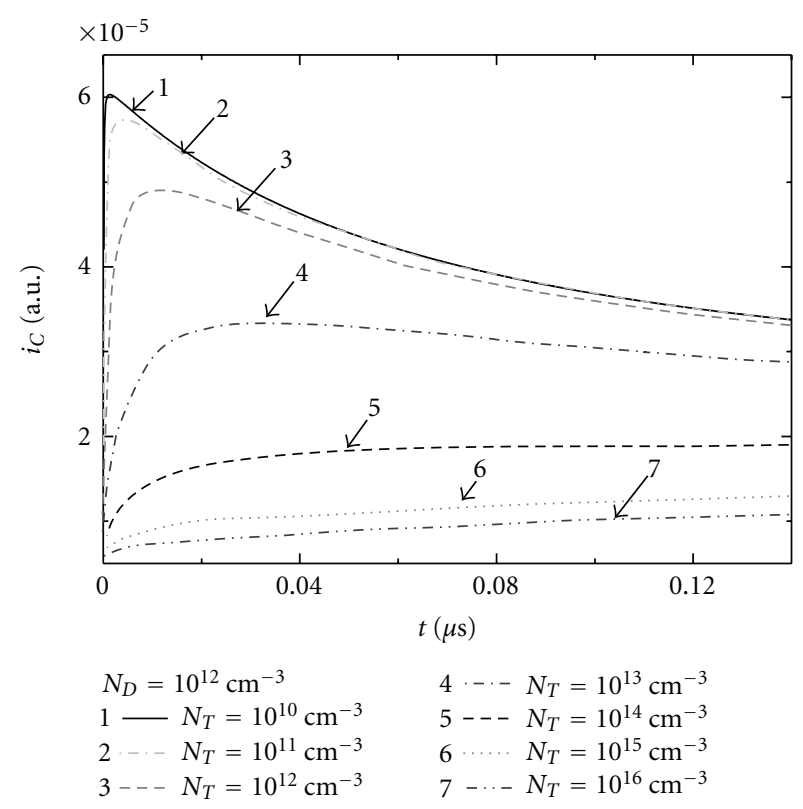

Figure 5: Comparison of the simulated charge extraction BELIV current transients varying density $\left(10^{10}-10^{16} \mathrm{~cm}^{-3}\right)$ of carrier capture centers in Si material with $N_{D}=10^{12} \mathrm{~cm}^{-3}$ doping-density.

$$
\begin{aligned}
& \left.+C_{\text {diffo }}\left(\exp \left(\frac{e U_{F}(t)}{k_{B} T}\right) \times\left(\frac{e U_{F}(t)}{k_{B} T}+1\right)-1\right)\right] \\
& +\frac{e n_{i} w_{0} S}{2 \tau_{R}}\left(1-\frac{U_{F}(t)}{U_{b i}}\right)^{1 / 2} \exp \left[\frac{e U_{F}(t)}{2 k_{B} T}\right] \\
& +i_{\text {diffo }}\left(\exp \left[\frac{e U_{F}(t)}{k_{B} T}\right]-1\right) .
\end{aligned}
$$

Here, additional symbols represent the following: $\tau_{R}=[1+$ $\left.2\left(n_{i} / n_{0}\right) \cosh \left(E_{T}-E_{i}\right)\right] / \sigma_{c} v_{T} N_{T} \approx 1 / \sigma_{c} v_{T} N_{T}, i_{\text {diff } 0}=$ $e S n_{i}^{2} D_{p}^{1 / 2} / N_{D} \tau_{p}^{1 / 2}$, and $C_{\text {diffo }}=i_{\text {diffo }} /(d U / d t) \cong i_{\text {diffo }} / A$. The storage (diffusion) capacitance is routinely assumed to be $C_{\text {diff }}=d\left(\int_{0}^{t} i_{\text {dif } 0}\left(\exp \left(e U\left(t^{\prime}\right) / k_{B} T\right)-1\right) d t\right) / d U$. At forward bias LIV, the initial stage of the BELIV transient is governed by a barrier capacitance current (first constituent in (10)), which rather slowly increases with $\left.U_{F}(t)\right)$. At $t \cong 0$, that is, for $e U_{F}(t) / k_{B} T \approx 0$, diffusion capacitance and diffusion current are close to zero. Thus, at low injection level regime, the barrier capacitance component prevails over the diffusion and recombination current constituents within initial stage of the current transient. The geometrical capacitance determines the initial step and capacitor like BELIV transient behavior, when a diode is fully depleted without external voltage. However, contrary to reverse biasing (1), where charge extraction causes a negative constituent $U_{R} d C_{b} / d U_{R}$, the barrier capacitance current component shows a positive derivative $U_{F} d C_{b} / d U_{F}>0$ for the forward-biased diode. This is determined by a denominator function within the first constituent of (10). The amplitude of the BELIV current, during rearward phase of BELIV current transient, exponentially increases due to $i_{\text {Cdif }}$ and $i_{\text {dif }}$ for the short LIV pulses
(Figure 6(a)). Thereby both the barrier capacitance $\left(i_{C F}(t)\right)$ charging current and the total current $\left(i_{\Sigma F}(t)\right)$ increase with LIV. Actual voltage drop on a diode $U_{F}(t)=A t-R_{L} i_{\Sigma} F(t)$ deviates from a linear increase, when $i_{\Sigma F}(t)$ is enhanced, even in the case when GLIV signal is perfect. This requires a solution of voltage sharing equation similar to that of (4). Then, the initial increase of $i_{C F}(t)$ is additionally modified by the $R C_{b 0}$ parameter of a circuit. Actually, only numerical simulations of time-dependent current $i_{F}(t)$ variations should be employed for the precise evaluations of barrier parameters at elevated voltages.

The simulated variations of the current components and of the total current (during LIV pulse) are presented in Figure 6(a). These transients have been simulated by assuming a long carrier lifetime approximation. The two componential $i_{\Sigma F}(t)=i_{C F}(t)+i_{\text {Cdif }}(t)$ current transients are clearly observed in Figure 6(a). The storage capacitance component dominates within $i_{\Sigma F}(t)$ during ulterior stages of a transient. Small amplitudes of $U_{F}<U_{b i}$ are preferential in simulations and experiments, in order to reduce a nonlinear sharing of voltage drops between the load resistor and the device under test.

The transients of composite (total) current simulated for two values of $U_{F}$, keeping the same pulse duration (i.e., varying ramp) and including the initial delay of $R C=20 \mathrm{~ns}$, are illustrated in Figure 6(b). It can be noticed that the initial amplitude and, especially, the rearward component of a transient is rather sensitive to the LIV pulse ramp. The experimental BELIV current transients obtained by varying the ramp of forward bias are illustrated in Figure 6(c). The qualitative changes of the amplitude and of the shape within BELIV transients, measured on Si diode irradiated with rather small fluence (Figure 6(c)), as a function of LIV voltage are in good agreement with those simulated (Figure 6(b)) using approximation of long carrier lifetimes.

For quantitative estimation of barrier characteristics, the combined analysis of height of the initial step of current for the reverse- $i_{R \Sigma}(0) \approx A C_{b 0}+i_{g}$ and for the forward- $i_{\Sigma F}(0)=$ $A C_{b 0}+\left(e n_{i} w_{0} / 2 \tau_{R}\right)$ biased diode is performed.

3.5. Profiling of Junctions. The characteristics discussed previously are inherent for the measured BELIV transients on structures with parallel-plate layers and electrodes. For electrodes of the perpendicular configuration, using a needle-tip electrode positioned on the cross-sectional boundary of a parallel-plate layered structure, the current spreading effect should be included. The spreading of current trajectories acts as a serial resistor $R_{S}$ introduced into BELIV measurement circuitry. For perpendicular geometry of electrodes an impact of the junction type at the needle-electrode is important.

The layered junction structures can be controlled by profiling of barrier charging currents for perpendicularly located electrodes using the BELIV technique. The boundary needletip electrode introduces several complications when using a simple evaluation of parameters. The spreading of currents from a nearly point contact appears even in the case when a single homogeneously doped layer is profiled. The simulated (using TCAD platform) distribution of potential and 


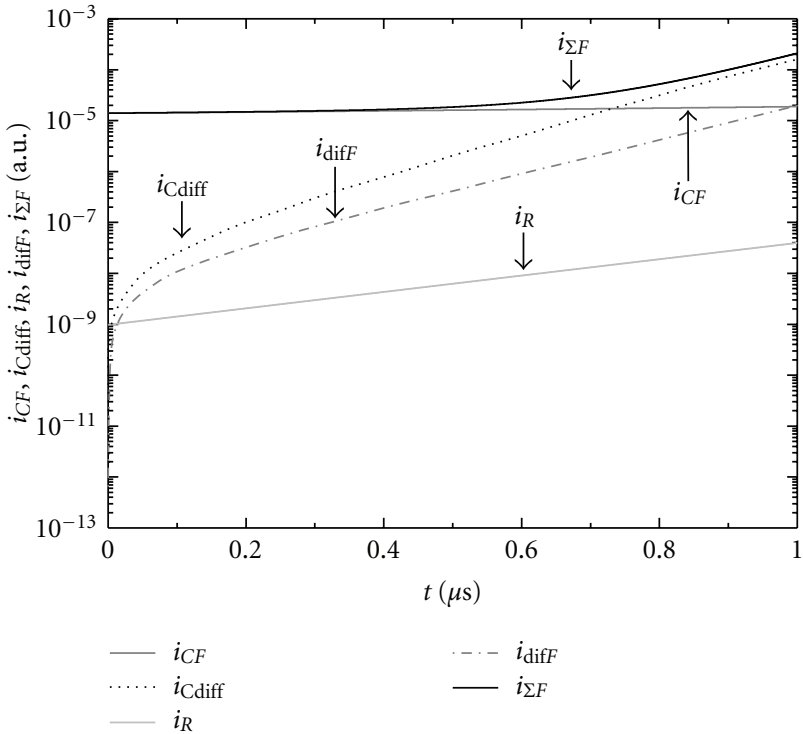

(a)

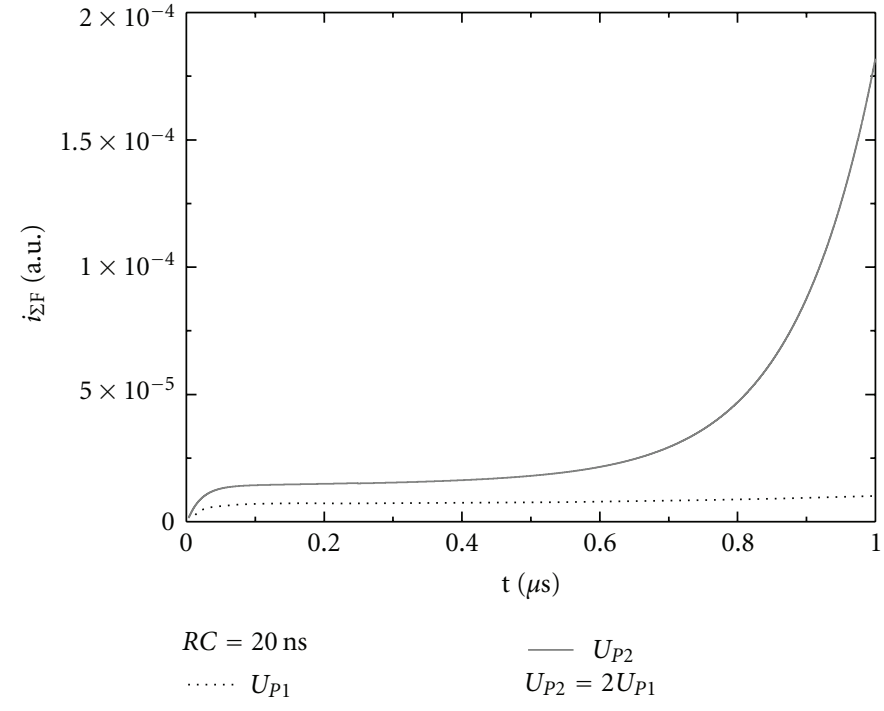

(b)

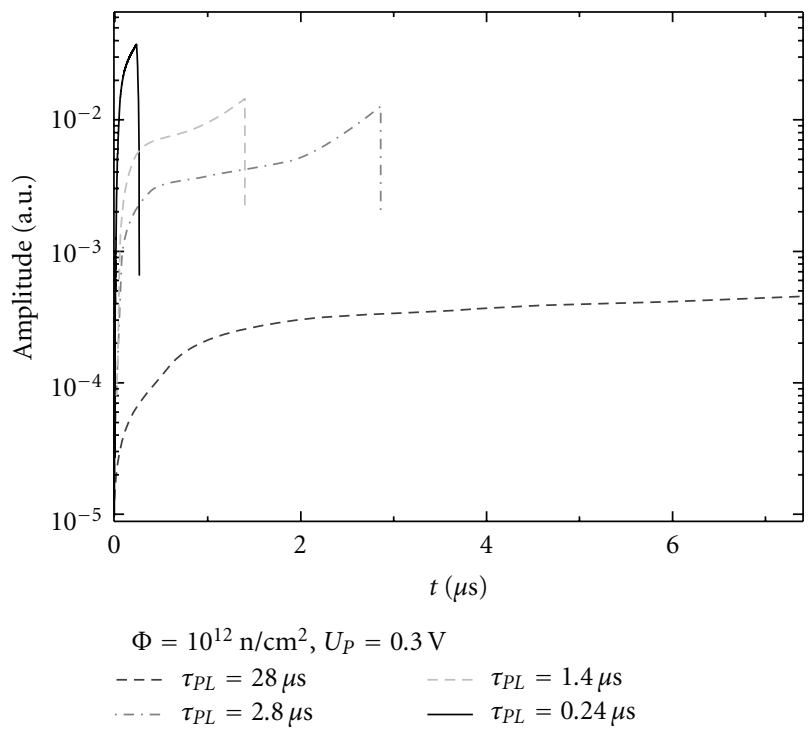

(c)

Figure 6: (a) Simulated total current (black solid) at forward LIV bias composed of barrier (grey solid) and of storage capacitance (dot) currents as well as of recombination (light grey solid) and of diffusion (dash-dot) currents. (b) The simulated BELIV transients varying $U_{P}$ of the forward LIV bias. (c) Experimental BELIV transients for forward- $\left(U_{F}\right)$ biased diode varying ramp $A$ of LIV pulses. The pulse peak amplitude $U_{P}=0.3 \mathrm{~V}$ was kept invariable while pulse duration was varied.

of current density is illustrated in Figures 7(a) and 7(b), respectively. Actually, the needle electrode induces a contact of varied surface area for a current flow. This is a reason for a spreading resistance $\left(R_{S_{\perp}}\right)$ dependent on probe location $y$ and on electrode diameter $a$, which can be expressed by the geometrical $(y, a, \Theta)$ and voltage $(U)$ parameters, as follows:

$$
R_{S_{\perp}}=\rho(U, y+a) \frac{l(y, \Theta)}{S(y, \Theta)}=\rho_{0}(y+a) \frac{l_{\mathrm{eff}}}{S_{\mathrm{eff}}}(y, t, U(t)) .
$$

Here, $\rho$ is a resistivity of material of the $i$-layer, and $\Theta$ is a geometrical width of a plate contact (assuming a square shape of this electrode). Measured resistivity $\rho$ depends on a probe position $y$ when material exhibits $\rho_{0}$ inhomogeneity. $\rho$ is dependent on voltage $U$ due to interface between the needle electrode and the material under test and due to a finite diffusion length of the injected excess carriers. The LIVinduced carriers $\Delta n_{0}$ modify conductivity of the material (with equilibrium carrier density $n_{0}$ ). However, a real probe does not provide an ideal ohmic contact. Thus, density of the injected excess carriers follows a characteristic of the forward biased Schottky barrier: $\Delta n=\kappa \Delta n_{0}\left[\exp \left(e U(t) / k_{B} T\right)-1\right]$. 


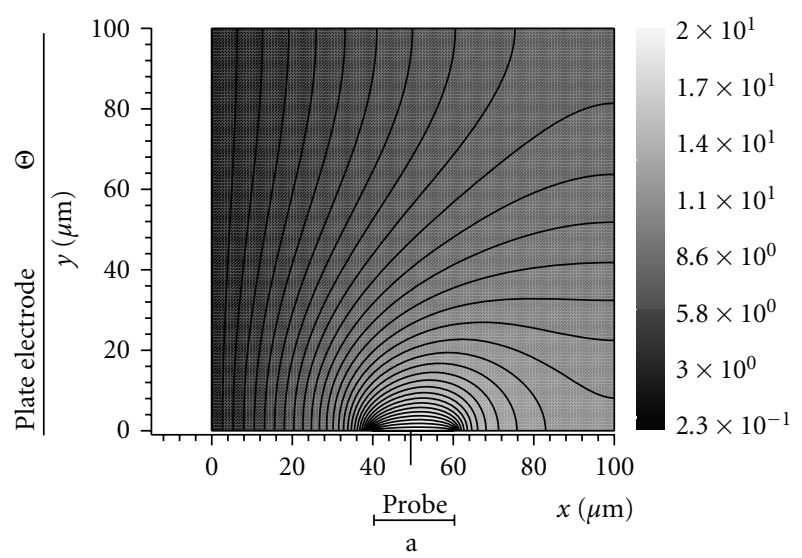

(a)

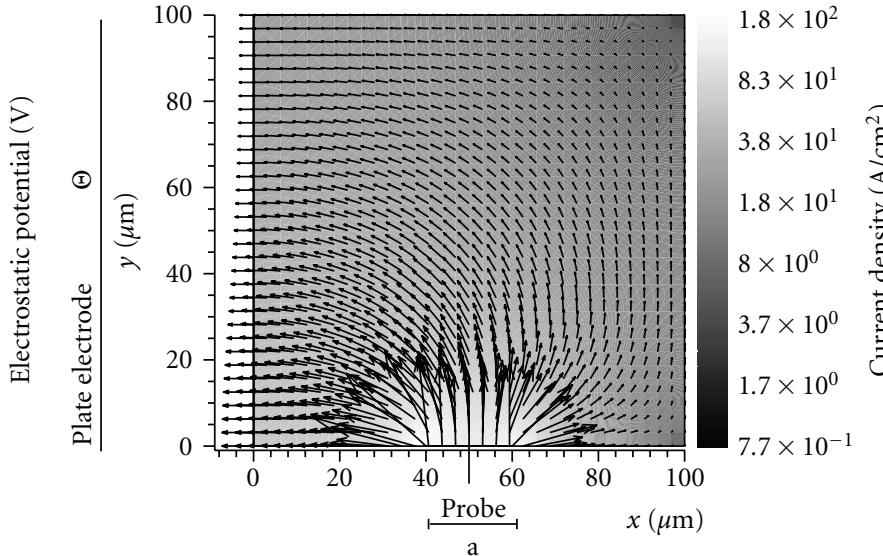

(b)

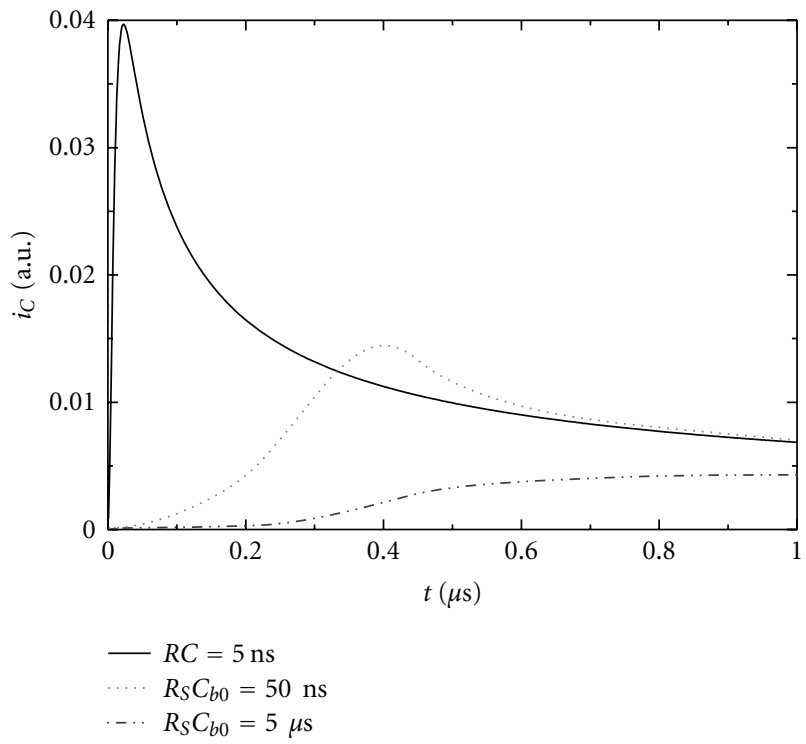

(c)

Figure 7: Simulated (by using TCAD platform, Alternative Solutions) distribution of potential (a) and of current density (b) for the perpendicularly located probes within a single layer. Values of potential are indicated by a white-gray-black bar in (a) Length of vectors shows simulated current density in (b). (c) Comparison of the simulated (at reverse bias) BELIV current $i_{C}(t)$ transient (for parallel-plate electrodes and for initial delay $R C=5 \mathrm{~ns}$ : solid grey curve) with those obtained for a needle-tip probe located on boundary of layered structure within an elevated resistivity material layer (behind the interface of the abrupt junction), when delay $R_{S} C_{b 0}$ (broken and black curves) is dependent on spreading resistance $R_{S \perp}$. The broken and black (solid) curves illustrate simulated transients for a BELIV response measured on $R_{L}=50 \Omega$ using a convolution integral with $R_{S} C_{b 0}$ values of $R_{S} C_{b 0}=5 \mathrm{~ns}$ (black solid), $50 \mathrm{~ns}$ (dot), and $5 \mu \mathrm{s}$ (dash-dot), respectively.

Summarizing both reasons, $\rho$ dependence on voltage and time (within BELIV current transient) can be expressed as follows:

$$
\begin{aligned}
\rho(U(t), y)=\rho_{0}(y+a)\left[1+\left(1+\frac{\mu_{p}}{\mu_{n}}\right) \frac{\kappa \Delta n_{0}}{n_{0}}\right. \\
\times \exp \left\{-\frac{\left(r-\mu_{p} E(r, t) t\right)^{2}}{4 D_{p} t}-\frac{t}{\tau_{R}}\right\} \\
\left.\times\left\langle\exp \left(\frac{e U(t)}{k_{B} T}\right)-1\right\rangle\right] \\
=\rho_{0}(y+a) F[r, t, E(r, t), U(t), D, \mu, \tau] .
\end{aligned}
$$

Here, $r$ is a length of a radius-vector for a definite point within a tested volume of material, $\mu_{n}$ and $\mu_{p}$ are mobilities of carriers, $D$ is carrier diffusion coefficient, $\tau_{R}$ is carrier recombination lifetime, $E(r, t)$ is electric field dependent on time and on spatial coordinate, and $\rho_{0}$ is an equilibrium resistivity of the material at $y$ point using a probe of $a$ diameter, $\kappa$ is a dimensionless (adjustable) factor to take into account of the proportionality between carrier density and current. $l_{\text {eff }} \sim$ $\int F d r$ is an effective length integrated over the current trajectories, and it should be actually integrated together with $F(r, t, U(t), \ldots)$ function. The same complication would appear in evaluation of effective surface area $S_{\text {eff }}$ for spread currents. Actually, separated simulation of $l_{\text {eff }}$ and of $S_{\text {eff }}$ is an 
incorrect procedure, as the currents spreading within a volume under test should be analyzed. Thus integrated (effective) ratio of $l_{\text {eff }} / S_{\text {eff }}$ (as a single parameter) can be only considered. For high symmetry of measurement configuration, as in common spreading resistance measurements, the approximation $l_{\text {eff }} / S_{\text {eff }}=1 / 2 a$ is widely accepted. Actually, rather low symmetry of the geometrical configuration does not allow simplifications in our case. Therefore, it is a complicated task for the numerical simulations, which also includes solution of a Poisson equation. Numerically simulated distributions of potential and currents for perpendicular geometry of asymmetric electrodes, emulating a configuration of our experimental regime, are illustrated in Figures $7(a)$ and $7(b)$.

It can be noticed in Figures 7(a) and 7(b) that concentration of electric field and of potential as well as of current density, respectively, is significantly enhanced at the probe edge. Actually, the strongest field is located at the beginning of probe. Thus, spatial resolution of profiling can be higher than that evaluated using a diameter $(a)$ of probe.

More attractive way, to evaluate the ratio of $l_{\text {eff }} / S_{\text {eff }}$, is a calibration procedure made by varying $U_{P}, \tau_{P L}$ (to find a regime close to the ohmic one) and a set of material samples combined with the definite probe and by attributing the calibrated current value to the peak amplitude of BELIV current transient.

Actually, monitoring of the correlated changes of LIV pulse and of current response enables one to control the ohmic regime for a single layer. Then, the current measured directly on a load resistor $R_{L}$ gives a value of $R_{S_{\perp}}$. Subsequently, variations of current values show $\Delta \mathrm{R}_{S, y(i+1)} \sim$ $R_{S, y(i)} l_{\mathrm{eff}(i+1)} / l_{\mathrm{eff}(i)}$ changes relatively to the previous probe location points.

Interface of a junction is clearly manifested within profiling scan by a crucial change of the BELIV transient shape and of peak amplitude, when needle-probe crosses this junction. The simulated variations of transients dependent on spreading resistance within an elevated resistivity layer are illustrated in Figure 7(c), for a needle probe located behind the interface. Barrier charging current is significantly less than an ohmic current within a large conductivity layer (e.g., either metallic electrode or $p^{+} / n^{+}$layers). Therefore, the peak amplitude crucially drops, while a shape of a transient changes from an LIV-pulse-like to that inherent for charge extraction current (Figure 7(c)).

Further fragment of the depth-profile of BELIV current transients, recorded relatively to a junction location, provides the additional characteristics for evaluation of $R_{S \perp}$ and for resolution of an impact of the deep centers within highresistivity layer. Impact of the deep traps can be resolved: either (i) as an appearance of a recess in the range of barrier capacitance charging peak when the traps filling process at depletion boundary (within $\lambda$-layer) ceases moving of this boundary (due to carrier extraction) or (ii) as a manifestation of a current minimum within a BELIV current transient with a current increment within the ulterior component of the transient due to an enhancement of generation current, as illustrated in Figure 3. The increased $R_{S \perp}$ determines a significant delay $\left(R_{S_{\perp}} C_{b 0}\right)$ of an initial BELIV current peak.
The barrier capacitance ascribed to the same plate electrode, employed for profiling, could be tested by measuring the BELIV current transient using the parallel-plate electrodes (when $R_{S \|}$ can be ignored). Then, delay time $R_{S \perp} C_{b 0}$, obtained from the profiling measurements, enables one to extract the $R_{S \perp}$ value.

The additional verifications of the electrode capability, as a carrier reservoir, are necessary in such a profiling measurement, to avoid current limitation due to quality of electrode. This can be performed by analysis of the amplitude of the current transient dependent on $U_{P}$ and by combining the reverse and forward LIV biasing regimes. The impact of generation current should be controlled by monitoring of the transient shape changes through varied pulse duration $\tau_{P L}$. The carrier capture characteristics can be examined by using additional steady-state bias illumination to vary filling of traps, in the case when the initial carrier extraction current peak appears to be deformed. The influence of spreading resistance and of serial resistance can be separated by combining the cross-sectional and parallel-plate measurement regimes on the same structure.

\section{Fluence, Temperature, and External Steady-State Bias-Dependent BELIV Characteristics in the Irradiated Si Diodes}

Variations of the charge extraction and injection BELIV currents as a function of voltage increase within LIV pulse are illustrated in Figure 8. These characteristics show clear dependence on neutron irradiation fluence.

For the reverse bias LIV pulses, it is clearly observed that the charge extraction current dominates at the relatively small fluences. This enables one to evaluate barrier parameters as $U_{b i}$ and $N_{\text {Def }}$ by employing the BELIV technique. The $N_{\text {Def }}$ seems to be the dominated factor, and $C_{b o}$ decreases with enhancement of fluence from $10^{12} \mathrm{n} / \mathrm{cm}^{2}$ to $10^{13} \mathrm{n} / \mathrm{cm}^{2}$; see Figure 8.

This can be explained by increment of density of compensation centers, located within a lower half of the band gap. This increment of density of compensation centers enhances the initial depletion width $w_{0}$ and, subsequently, decreases value of the $C_{b o}$. However, starting from fluences of $10^{14} \mathrm{n} /$ $\mathrm{cm}^{2}$, the generation current becomes the prevailing component of a transient at reverse bias voltage. Thus, BELIV current transient exhibits a trapezoid shape starting from the initial step in diodes irradiated with fluence of $10^{14} \mathrm{n} / \mathrm{cm}^{2}$. Close values of $C_{b o}$ and the trapezoid shape of transient are obtained for $10^{16} \mathrm{~cm}^{-2}$. The generation current also determines a pedestal and compensates a reduction of $C_{b 0}$. Therefore, comparison of the initial step for the reverse- and forward-biased diodes should be employed to exclude this pedestal. The initial step in BELIV current transients for the forward-biased diode (when $C_{b o}$ prevails) should coincide with that for a reverse-biased diode. It is clear from comparison of these transients that $C_{b o}$ values are of close magnitude for $10^{14} \mathrm{n} / \mathrm{cm}^{2}$-irradiated diodes (including difference in $U_{P}$ ramp). It can be noticed that in a reverse-biased diode, 


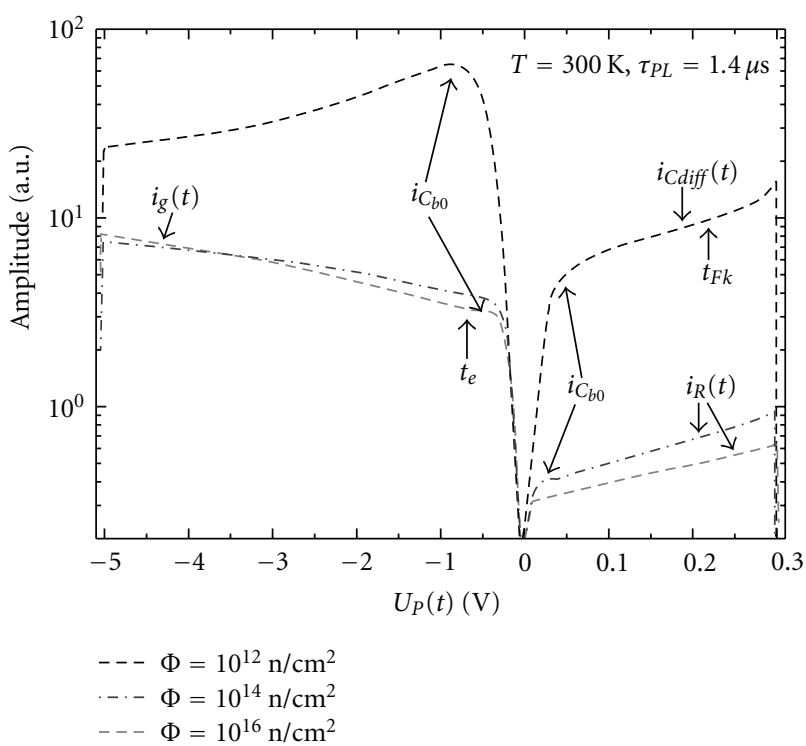

FIGURE 8: Variations of barrier and diffusion capacitance charging currents dependent on irradiation fluence as a function of voltage increase within LIV pulse in Si pin detector, at the same LIV parameters.

generation current increases with fluence $\Phi$, and, at $\Phi=$ $10^{16} \mathrm{n} / \mathrm{cm}^{2}$, it exceeds that value measured for $10^{14} \mathrm{n} / \mathrm{cm}^{2}$ irradiated diode. The amplitude of the initial step also decreases for the forward-biased diode. This correlates with results obtained for the reverse-biased diode. Variations of these characteristics with irradiation fluence can be explained by approaching of value of the initial barrier capacitance to that of geometrical one. It is worth noting that impact of the storage capacitance can be observed only in lightly $\left(10^{12} \mathrm{n} / \mathrm{cm}^{2}\right)$ irradiated diode at rather small forward biasing voltage.

For a forward-biased diode (right side in Figure 8), the barrier capacitance prevails in the initial stages of transient measured on a diode irradiated with relatively small fluence. Then reduction of stored charge and of diffusion length of the injected carriers leads to a decrease of forward current within transient, if carrier lifetime becomes shorter than that of LIV pulse. This result is in excellent agreement with carrier lifetime values measured directly using a microwave probed photoconductivity transients (MW-PCD) [10] for the same samples. Reduction of the $C_{b o}$ is determined by an enhancement of the density of compensating centers, and it is reduced to $C_{\text {geom }}$ when equilibrium depletion width approaches to a sample thickness.

For the noncapsulated diode structures, trap-determined modifications of the BELIV current transients of $i_{C}(t)$ can be suppressed through the priming of the trap filling by infrared (IR) continuous wave illumination. Additionally, the initial component of BELIV current transient can be manipulated by a pedestal of varied polarity of the dc voltage together with an LIV pulse. Variations of the mentioned external factors can be combined with temperature variations to modify filling of the traps.

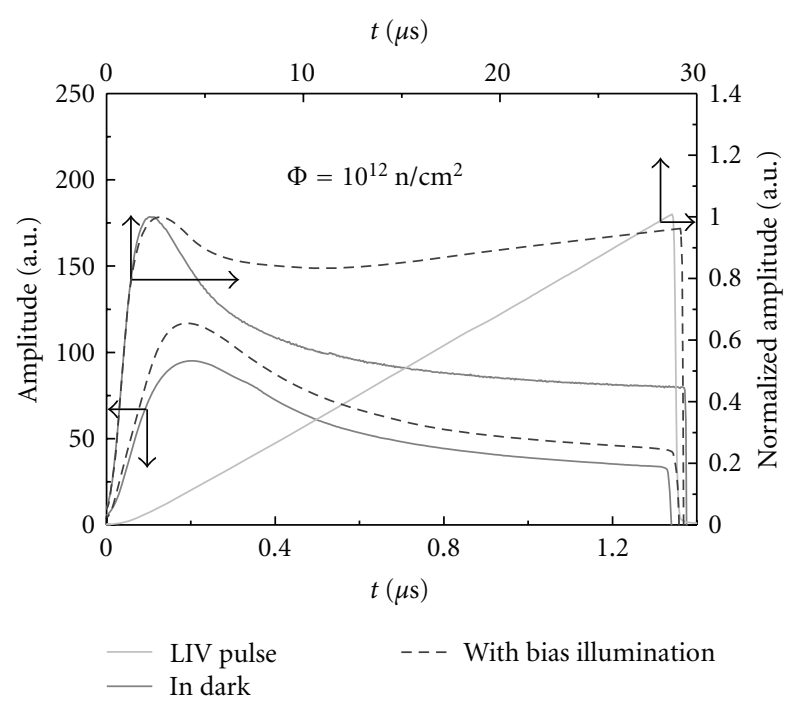

FIGURE 9: Bias illumination- (BI-) dependent charge extraction current transients (as measured: solid curves; normalized to a peak amplitude: broken curves) measured on the same irradiated diode at a fixed reverse $\left(U_{R}\right)$ voltage of LIV pulses.

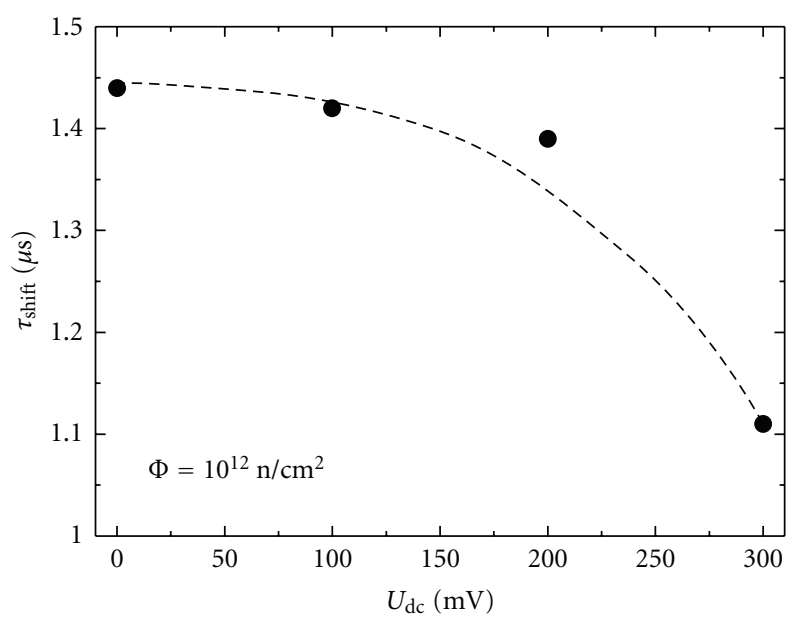

FIGURE 10: A shift of the barrier capacitance charging current peak position within transients recorded for reverse-biased LIVdependent on forward dc voltage bias pedestal.

In Figure 9, variations of BELIV current transients measured with and without bias illumination (BI) are presented. The primary steady-state illumination modifies the initial filling of traps for electrons and holes. It has been revealed in our investigations on spectral efficiency of bias illumination that spectral range of the interband absorption $(1.12 \mathrm{eV}$ for $\mathrm{Si}$ at room temperature) is optimal for primary filling of traps by IR light. Depending on duration of LIV pulses and on density of traps, the time scale of carrier generation processes can be highlighted, as illustrated in Figure 9. The absolute value of the initial amplitude of BELIV current transient for a reverse-biased diode increases proportionally to the intensity of the bias illumination. Simultaneously, a component of generation current within ulterior stages of transient is 


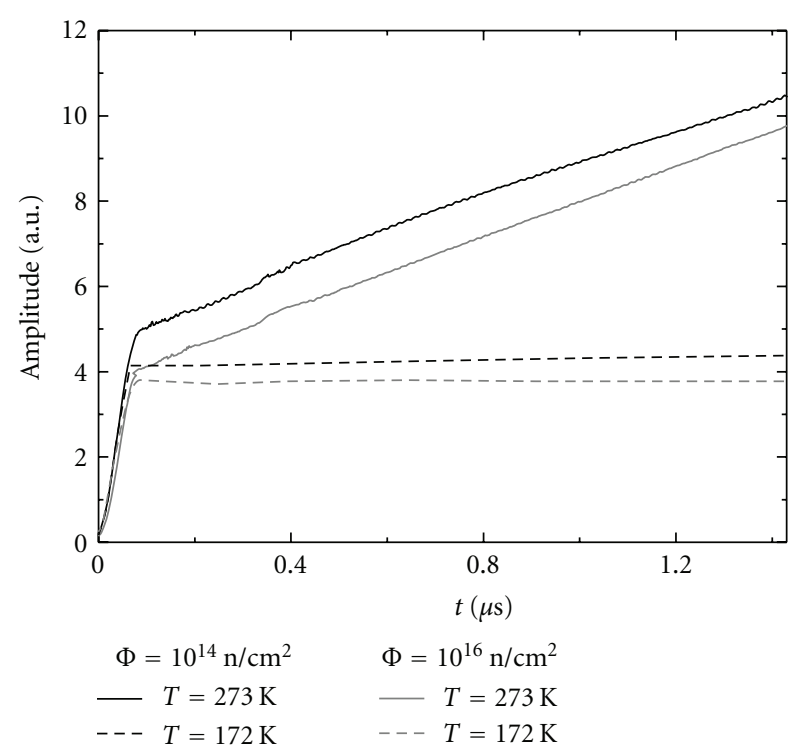

(a)

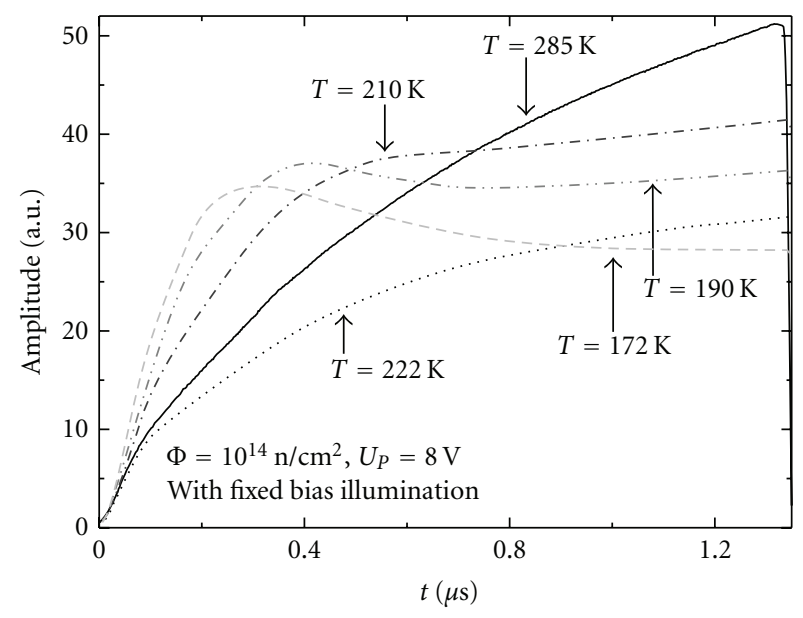

(b)

Figure 11: (a) Temperature-dependent variations of BELIV current transients in heavily $\left(10^{14}\right.$ and $\left.10^{16} \mathrm{n} / \mathrm{cm}^{2}\right)$ irradiated $\mathrm{Si}$ pin diode. (b) Temperature and bias illumination-dependent variations of the BELIV current transients.

increased with BI. Increment of generation current is more obvious for the normalized (to the amplitude of the initial step) transients, shown in Figure 9. This result clearly proves that generation current is caused by several traps characterized by a wide spectrum of levels within the upper half of band gap, as simulated in Figure 4. Calibration measurements of the density of the absorbed BI quanta enable one to evaluate density of capture centers, for which the saturation of the amplitude of BELIV current is reached. For rather long LIV pulse, duration of which corresponds to thermal emission time scale (characteristic for a definite sample), the generation current becomes dominant within the rearward component of a transient. BELIV current transients for reverse bias LIV pulses can be manipulated by the external light only in diodes irradiated by rather moderate fluences, $\leq 10^{13} \mathrm{n} / \mathrm{cm}^{2}$, at room temperature.
Complementarily, filling of the minority carrier trap can be implemented by dc forward voltage pedestal combined with BELIV current transient measurements using the reverse-biased LIV pulses. Injection of minority carriers enables one to eliminate minority carrier traps (those seem to be efficient as the compensation centers within a lower half of band gap), and to regenerate barrier capacitance, as shown in Figure 10. Enhancement of a forward dc voltage pedestal leads to filling of these traps. This determines an increase of the BELIV current signal amplitude and a shift of the peak to the initial time instants within a transient, as illustrated in Figure 10. Calibration of the injected density of minority carriers, when possible, and fixation of a reached saturation level for the amplitude of BELIV current transients can be exploited for evaluation of the density of the compensation centers. However, a suppression of compensating centers at room temperature has been reached in our experiments only for moderately irradiated $\left(\leq 10^{14} \mathrm{n} / \mathrm{cm}^{2}\right)$ diodes.

An impact of radiation induced generation centers can be also suppressed by reduction of temperature, as carrier emission lifetime increases about exponentially with reduction of temperature. The temperature-dependent BELIV current transients are illustrated in Figure 11(a) for a heavily irradiated $\left(10^{14}\right.$ and $\left.10^{16} \mathrm{~cm}^{-2}\right)$ and reverse-biased pin diode. It is clearly seen that the component of the carrier generation current decreases with reduction of temperature. Then BELIV current transient approaches to that inherent for dielectric capacitor, at $172 \mathrm{~K}$. Thus, heavily irradiated diode is fully depleted at equilibrium, although at room temperature this capacitor-inherent characteristic is masked by the large generation current component.

With reduction of temperature, which leads to a consequent increment of carrier emission time and to a decrease of density of the empty capture-emission centers, the steadystate bias illumination becomes sufficient for suppression of the carrier capture centers. It can be seen in Figure 11(b) that the barrier capacitance in moderately irradiated $\left(\leq 10^{14} \mathrm{n} /\right.$ $\mathrm{cm}^{2}$ ) diodes restores to values inherent for lightly irradiated diodes when a combined conditioning made by the temperature lowering and additional illumination is applied. However, neither steady-state biasing by dc forward voltage nor continuous wave illumination is sufficient to suppress charger compensation and carrier capture/generation centers in heavily irradiated $\left(>10^{15} \mathrm{n} / \mathrm{cm}^{2}\right)$ diodes.

There are several options for evaluation of the diode parameters by using BELIV technique. The fluence dependent variations of the parameters of $C_{b 0}, U_{b i}, \tau_{g}, N_{\text {Def }}$, and $N_{T}$ can be examined by using peculiar points and segments on the BELIV current transients, exploiting approximations expressed by (1)-(3),(6)-(8), and (10), when rather small voltages are applied. This enables to ignore the nonlinear voltage sharing between $R_{L}-C_{b 0}$ elements of circuit. The numerical simulations, using approximations described in (1)-(3), (4)(8), (10) and $U_{c}(t)=U_{P}(t)-i_{\sum}(t)\left(R_{L}+R_{s||}(t)\right)$, are inevitable when both high precision and wide range of voltages are desirable. Here, $R_{s||}(t)$ represents a serial bulk resistance which can be approximated as $R_{s \|}(t)=(d-$ $w(t)) / \operatorname{Se} \mu n(t)$. 


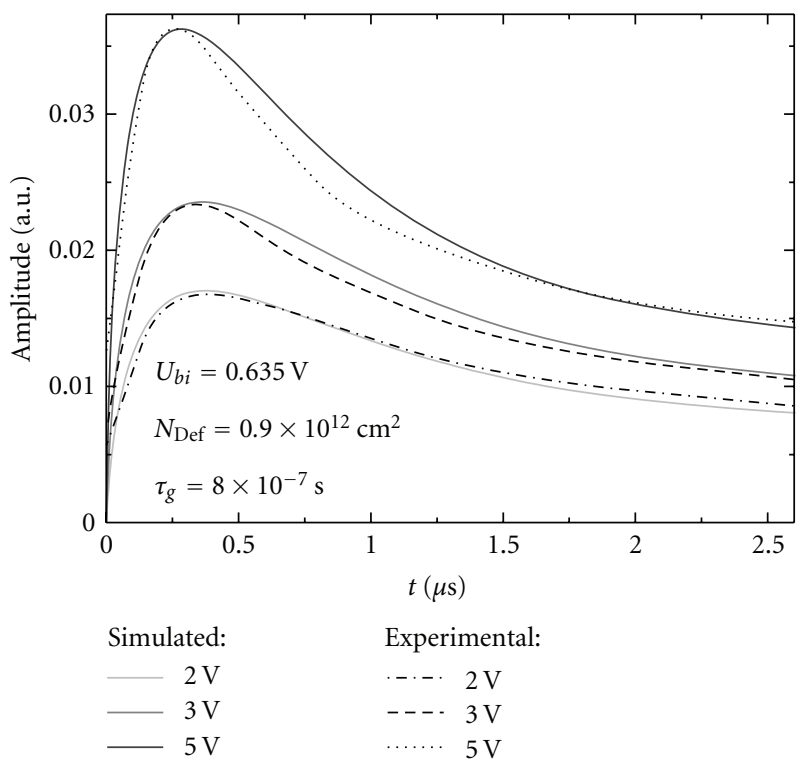

(a)

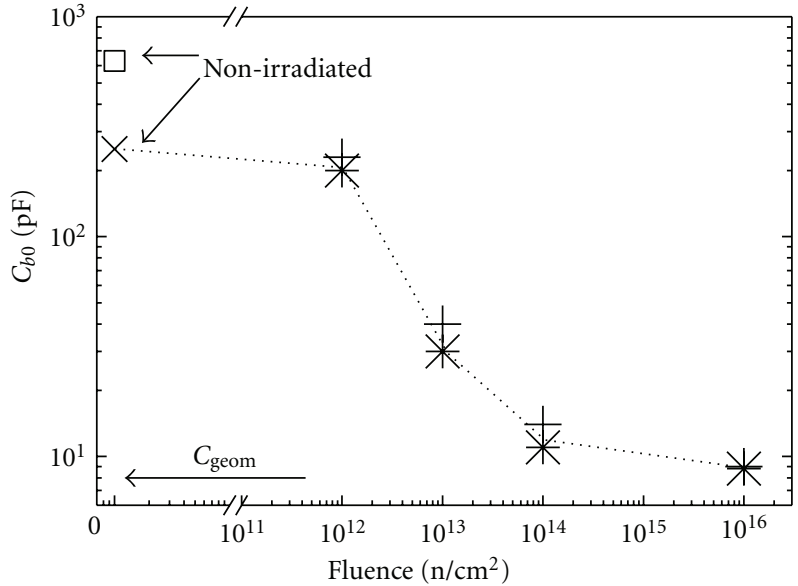

Industrial non-irradiated diode $\quad C_{b 0}$ : BELIV, $\tau_{P L}=1.4 \mu \mathrm{s}$ $\rho \sim 25 \Omega \mathrm{cm}$

$\square$ Reverse bias

MCZ non-irradiated diode $\rho \sim 1 \mathrm{~K} \Omega \mathrm{cm}$

$X$ Reverse bias
Neutron irradiated diodes

* Reverse bias

+ Forward bias

(b)

Figure 12: (a) A family of transients, measured by varying LIV pulse ramp, fitted by simulated ones, when minimum for least-square deviations between simulated and experimental transients has been obtained. The set of parameters $U_{b i}, N_{\text {Def }}$ and $\tau_{g}$ has been simultaneously extracted by this fitting procedure. The extracted values are denoted within a legend. (b) Comparison of the fluence dependent variations of the barrier capacitance $C_{b 0}$ measured by BELIV technique employing both reverse (stars) and forward (crosses) LIV pulses at $T=300 \mathrm{~K}$. Values of $C_{b 0}$ obtained for nonirradiated Si diodes of different technology are also shown in (b).

An illustration of the fitting procedure by numerical simulations, applied to diode irradiated with $\Phi=10^{12} \mathrm{~cm}^{-2}$, is presented in Figure 12(a). To increase precision and to reduce an impact of the nonideality of LIV pulses, a family of transients measured by varying LIV pulse ramp $(A)$ have been simultaneously fitted with simulated ones (Figure 12(a)). Here, extraction of parameters had been controlled by a nonlinear-least-square (NLS) algorithm. A set of parameters of the $U_{b i}, N_{D}$, and $\tau_{g}$ have been either kept invariable or simultaneously altered for all the transients within a family of curves. The LIV pulse parameters $U_{P}$ and $d U_{p} / d t$ had been taken from experimental measurements within this fitting technique. The extracted parameters obtained from the best fit between experimental and simulated transients for NLS minimum are shown in the legend of Figure 12(a).

The extracted parameter $C_{b 0}$ as a function of irradiation fluence is shown in Figure 12(b). The initial barrier capacitance $C_{b 0}$ evaluated by both reverse and forward LIV pulses coincides within measurement errors (those not exceed the size of symbols in Figure 12(b)). The $C_{b 0}-\Phi$ characteristic indicates a clear decrease of absolute $C_{b 0}$ values due to outspreading of depletion width. The decrease of $C_{b 0}$ is caused by an enhancement of $w_{0}$ due to a reduction of $n_{0}$. Radiationinduced defects are fast traps that reduce the effective density of dopants $N_{\text {Def }}$ by formation of compensating centers. Consequently, a barrier $U_{b i}$ is modified. The detrapped charge leads to an increase of generation current component when trap density is significantly enhanced with fluence.

\section{Profiling of Junction Location in Solar-Cell Structures}

In order to verify whether the BELIV technique is proper to resolve rather thin layers, the solar-cell tentative $n^{+}-p$ structures containing $1-4 \mu \mathrm{m}$ thick $n^{+}$and metallization layers have been tested. Depth distribution of the amplitude scanned on the solar-cell structures is illustrated in Figure 13. In the insets of Figure 13, the inherent BELIV current transients, associated with different layers, are shown. Variations of the peak amplitudes enabled us to clearly resolve an interface location for a solar-cell structure.

Comparison of the profiles scanned on metalized and nonmetalized solar cells (Figure 13) enabled us to distinguish the top $4 \mu \mathrm{m}$ thick $n^{+}$layer and the metallic electrode. Here, only fragments $(0-15 \mu \mathrm{m})$ of the scanned profiles nearby the junction interface are shown. The profiles are plotted starting from the surface of the structure. In the case of the metal electrode deposited on heavily doped layer, the transient repeats LIV pulse shape over the length of a sum of the $d_{n+}+$ $d_{\text {metal electrode }}$ thicknesses. Thickness of $n^{+}$layer is consequently evaluated to be $\sim 1 \mu \mathrm{m}$ for a sample SC1 and $\sim 4 \mu \mathrm{m}$ for a sample SC2, respectively. The $1.25 \mu \mathrm{m}$ scanning step was kept in the latter profiling measurements. The main errors in evaluation of $d_{n+}$ appear due to inhomogeneity of thickness of metallization layer. The reduction of an area of the main junction plate electrode, for the sample SC2, leads to a decrease of a barrier capacitance and, consequently, to 

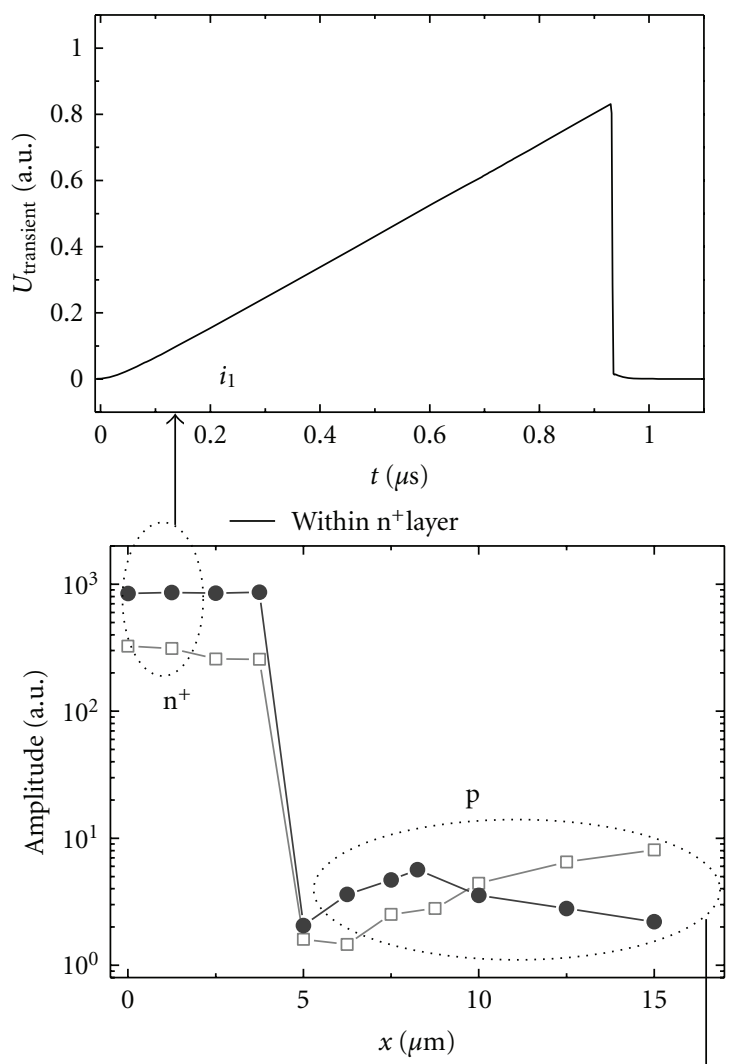

Completely metallized electrode surface

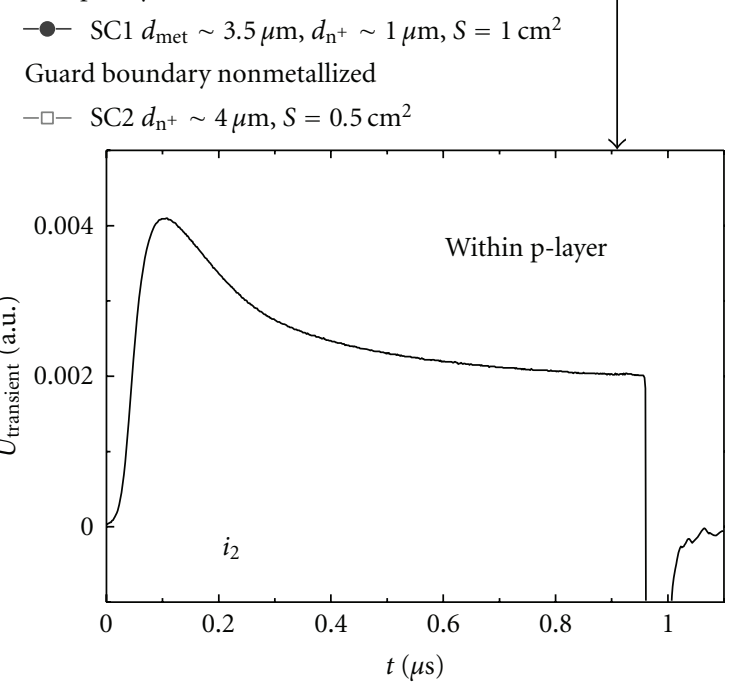

Figure 13: Profiles of the BELIV current amplitude in the solarcell structure containing different (metallization $/ n^{+}$) thickness and area of electrode. The shape of registered current transients within respective depth is shown in the insets.

a decrement of the BELIV current peak, relatively to SC1 sample. The outspread of the interfacial depth, with inherent increase of BELIV current amplitude (decrease of $R_{S||}$ and enhancement of the effective doping density), can be deduced within profiles, presented in Figure 13, going towards bulk of the $p$-layer.

Thus, the BELIV profiling technique is even suitable for scans on rather thin-layered structures using a rather wide

( $a \approx 10 \mu \mathrm{m})$ probe. As can be noticed in Figures 7(a) and 7(b) for simulated potential and spread of currents, the enhanced potential and current density appear at the front edge of the probe. Therefore, it is confirmed that resolution of scanning can be significantly better than width $(a)$ of a probe.

Examination of a rearward component in the illustrated BELIV transient (bottom inset of Figure 13) revealed an impact of the space charge generation current in the solar-cell structures. This has been highlighted by increasing an LIV pulse duration to match the time scale for carrier thermal emission. A significant impact of carrier generation processes in time scale exceeding $10 \mu$ s has been corroborated by the DLTS measurements on the same structures of Si solar cells, when metal impurities ascribed traps were identified.

\section{Summary}

In summary, the presented BELIV technique is a tool for fast evaluation of barrier parameters in the junction structures. This technique has been approved on different structures, and it is suitable for examination of the structures of irradiated particle detectors, pin-diodes, and solar-cells. Variations of the extracted parameters of $\tau_{R}$ and $\tau_{g}$ as a function of irradiation fluence are in good agreement with those measured by microwave probed photoconductivity transient technique on the same samples. Temperature-dependent variations of the $\tau_{g}(T)$ and $\tau_{R}(T)$ parameters, when $\tau_{g}$-associated and $\tau_{R}$ - ascribed current components are distinguishable within BELIV current transients can be applied for spectroscopy of deep levels. As usually, the latter components are dominant in heavily irradiated diodes. Thus, BELIV technique can be a useful extension of transient techniques for spectral analysis of deep levels.

The BELIV-pulsed technique enables one to clarify a few significant aspects: to identify charge extraction regime and to estimate barrier capacitance, to clarify competition between barrier capacitance $\left(i_{C}\right)$ and generation $\left(i_{g}\right)$ currents, and to clarify full-depletion state for heavily irradiated diodes. It has been shown that the built-in full-depletion (insulating state) is inherent for Si diodes doped with $10^{12} \mathrm{~cm}^{-3}$ donors density and irradiated with hadron fluences above $10^{14} \mathrm{~cm}^{-2}$.

The presented BELIV technique is applicable to fast estimation of actual location of barriers, of doping profiles, and of the deep traps within layered junction structures. This technique has been applied and appeared to be suitable for examination of pin-diodes and solar-cell structures. Depth variations of the amplitude and duration of the BELIV current transients and of the shape of these transients are in good agreement with such characteristics evaluated by using common $R_{S||}$ profiling instruments. The parameters of barrier capacitance, of density of the effective doping, and of space charge generation current extracted from the characteristics of BELIV current transients are in excellent agreement with those determined by DLTS techniques on the same samples. BELIV technique can be employed as a fast tool to obtain indications on existence of deep centers within definite layers for more detail identification of these traps by using the DLTS spectroscopy. 


\section{Acknowledgments}

G. Kramberger is appreciated for the neutro irradiations at TRIGA reactor. This research was funded by a grant MIP054/2011 from the Research Council of Lithuania.

\section{References}

[1] http://rd50.web.cern.ch/rd50/.

[2] M. Huhtinen, "Simulation of non-ionising energy loss and defect formation in silicon," Nuclear Instruments and Methods in Physics Research, Section A, vol. 491, no. 1-2, pp. 194-215, 2002.

[3] P. F. Ermolov, D. E. Karmanov, A. K. Leflat, V. M. Manankov, M. M. Merkin, and E. K. Shabalina, "Neutron-irradiationinduced effects caused by divacancy clusters with a tetravacancy core in float-zone silicon," Semiconductors, vol. 36, no. 10 , pp. 1114-1122, 2002.

[4] J. C. Vickerman and I. S. Gilmore, Surface Analysis-The Principal Techniques, John Wiley and Sons, Chichester, UK, 2nd edition, 2009.

[5] S. Kalinin and A. Gruverman, Scanning Probe Microscopy, vol. 1, Springer, New York, NY, USA, 2007.

[6] X. Ou, P. D. Kanungo, R. Kögler et al., "Carrier profiling of individual si nanowires by scanning spreading resistance microscopy," Nano Letters, vol. 10, no. 1, pp. 171-175, 2010.

[7] D. K. Schroder, Semiconductor Material and Device Characterization, John Wiley and Sons, Hoboken, NJ, USA, 3rd edition, 2006.

[8] P. Blood and J. W. Orton, The Electrical Characterization of Semiconductors: Majority Carriers and Electron State, Academic Press, New York, NY, USA, 1992.

[9] S. M. Sze, Physics of Semiconductor Devices, John Wiley and Sons, New York, NY, USA, 1981.

[10] E. Gaubas, A. Uleckas, and J. Vaitkus, "Spectroscopy of neutron irradiation induced deep levels in silicon by microwave probed photoconductivity transients," Nuclear Instruments and Methods in Physics Research, Section A, vol. 607, no. 1, pp. 92-94, 2009. 

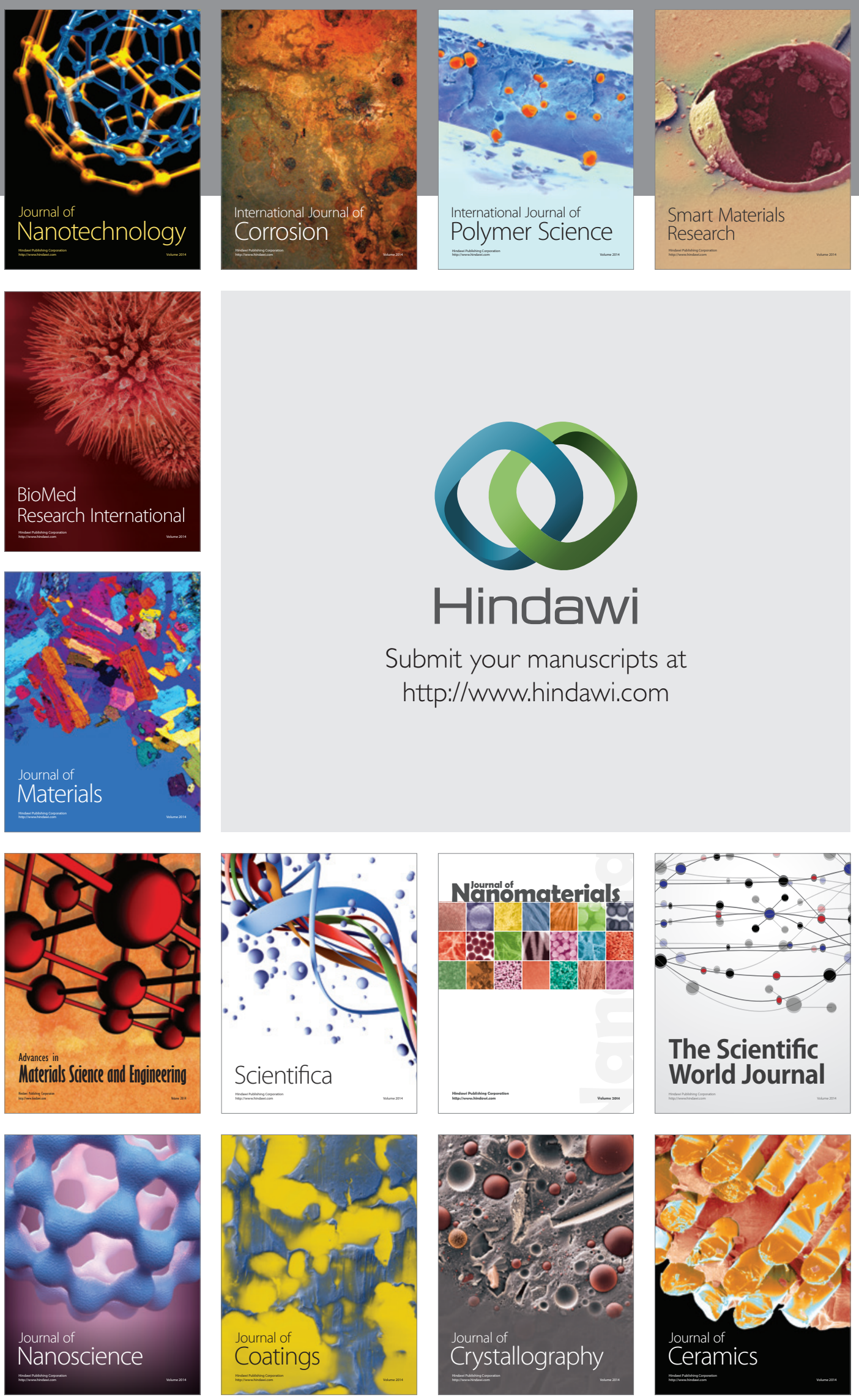

The Scientific World Journal

Submit your manuscripts at

http://www.hindawi.com

\section{World Journal}

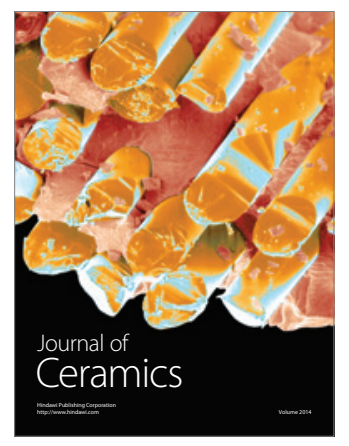

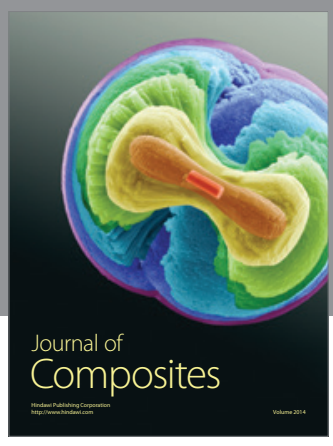
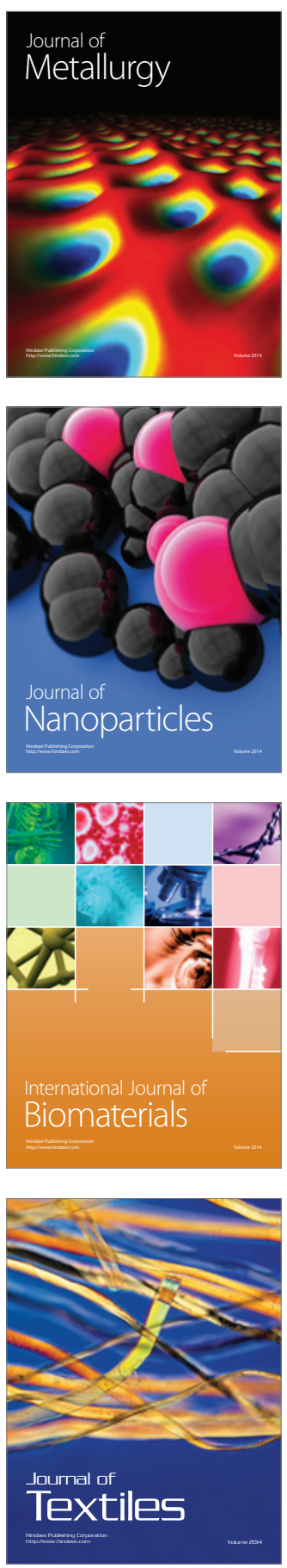